\title{
Local and substrate-specific S-palmitoylation determines subcellular localization of Gao
}

Gonzalo P. Solis ${ }^{1, *}$, Jana Valnohova ${ }^{1}$, Cecilia Alvarez ${ }^{2}$ and Vladimir L. Katanaev ${ }^{1,3, *}$

${ }^{1}$ Translational Research Center in Oncohaematology, Department of Cell Physiology and Metabolism, Faculty of Medicine, University of Geneva, 1211 Geneva, Switzerland;

${ }^{2}$ Centro de Investigaciones en Bioquímica Clínica e Inmunología (CIBICI-CONICET). Departamento de Bioquímica Clínica, Facultad de Ciencias Químicas, Universidad Nacional de Córdoba, Córdoba, Argentina;

${ }^{3}$ School of Biomedicine, Far Eastern Federal University, Vladivostok 690090, Russia; *Authors for correspondence: gonzalo.solis@unige.ch; vladimir.katanaev@unige.ch.

\section{ABSTRACT}

Peripheral membrane proteins (PMPs) associate with cellular membranes through posttranslational modifications like S-palmitoylation. The Golgi apparatus is generally viewed as the transitory station where palmitoyl acyltransferases (PATs) modify PMPs, which are then transported to their ultimate destinations such as plasma membrane (PM); little substrate specificity among the many PATs has been determined. Here we describe inherent partitioning of Gao - a-subunit of heterotrimeric Go-proteins - to PM and Golgi, independent from Golgi-to-PM transport. A minimal code within the Gao N-terminus governs the compartmentalization and re-coding produces G-protein versions with shifted localization. We established the SpONM (S-palmitoylation at the outer nuclear membrane) assay to probe substrate specificity of PATs in intact cells. With this assay, we showed that PATs localizing to different membrane compartments display remarkable substrate selectivity, which is the basis for specific PMP compartmentalization. Our findings uncover the fundamental mechanism governing protein localization and establish the basis for innovative drug discovery. 


\section{INTRODUCTION}

G protein-coupled receptors (GPCRs) and their immediate transducers - the heterotrimeric $G$ proteins - have been subjected to intensive scrutiny for decades, primarily due to their pivotal roles in innumerable physiological and pathological processes ${ }^{1,2}$. Heterotrimeric $G$ proteins are composed of $\mathrm{G} \alpha, \mathrm{G} \beta$ and $\mathrm{Gy}$ subunits. The $\mathrm{G} \alpha$ subunit is loaded with either GDP or GTP; the G $\beta$ and Gy subunits form a constitutive heterodimer that reversibly binds to Ga. GPCRs directly interact with heterotrimeric $G$ proteins on the cytosolic surface of the membrane. Upon activation, GPCRs act as exchange factors to enhance the release of GDP from Ga, leading to the binding of GTP and the activation of the Ga subunit. Subsequently, activated $\mathrm{G} \alpha$ dissociates from the receptor and the $\mathrm{G} \beta \mathrm{y}$ heterodimer, and the free subunits are competent to interact with downstream targets ${ }^{3}$.

G protein activation via GPCRs has long been thought to occur exclusively at the plasma membrane (PM). Most recently, however, considerable experimental evidence has accumulated supporting the notion that GPCRs can activate Ga subunits on the Golgi and other compartments ${ }^{4}$. Analogously, activation of the KDEL receptor (KDELR) by cargo from the endoplasmic reticulum (ER) was shown to trigger signal cascades via Gas and $\mathrm{Gaq} / 11$, with KDELR acting as a non-canonical GPCR at the Golgi ${ }^{5}$. Our own work showed that KDELR also binds and activates monomeric Gao, which in turn enhances the Golgi-to-PM trafficking via small Rab GTPases ${ }^{6}$. Therefore, the subcellular compartmentalization of $\mathrm{Ga}$ subunits appears to be of fundamental relevance for their functions. How Ga subunit compartmentalization is achieved and controlled, however, remains poorly understood.

Ga subunits are grouped into four subfamilies based on sequence and functional similarity: Gas, Gai, Gaq/11 and Ga12/13³. All Ga subunits bind to membranes via fatty acid modifications at the $\mathrm{N}$-terminus, i.e. N-myristoylation and S-palmitoylation? ${ }^{7}$. While the majority of $\mathrm{Ga}$ subunits are single-palmitoylated, Gao and other members of the Gai subfamily are dual-lipidated. N-myristolation occurs co-translationally and results in the attachment of a 14-carbon saturated fatty acid (myristate) to the N-terminal Gly via a stable amide bond ${ }^{8}$. S-palmitoylation on the other hand occurs post-translationally and results in the attachment of a 16-carbon saturated fatty acid (palmitate) to a Cys residue through a reversible thioester linkage ${ }^{9}$. In vertebrates, $\mathrm{N}$-myristolation is catalyzed by two closely related N-myristoyltransferases (NMT1 and NMT2) whose substrate specificities have been intensively studied in recent years ${ }^{10}$. Intracellular S-palmitoylation is catalyzed by a zincfinger Asp-His-His-Cys domain-containing ( $\mathrm{ZDHHC}$ ) family of palmitoyl acyltransferases (PATs) $^{11}$. There is up to $24 \mathrm{zDHHCs}$ described in mammals; opposite to NMTs, their 
substrate specificities are far from being well understood, although substantial advance has been made lately ${ }^{12}$.

S-palmitoylation of peripheral membrane proteins (PMPs), including Ga subunits, was shown to occur exclusively at the Golgi, where palmitoylated proteins are subsequently transported to the $\mathrm{PM}^{13}$. In recent years, however, cumulative data has emerged indicating that some PMPs might undergo local S-palmitoylation on their target compartments, namely the PM or $E R^{14,15}$. These opposing views appear difficult to reconcile primarily due to the lack of experimental methods allowing visualization of S-palmitoylation in intact cells.

Here, we defined the critical parameters that govern Gao N-myristolation, S-palmitoylation and subcellular compartmentalization. By engineering a system that allows the ectopic localization of $\mathrm{ZDHHCs}$ to the outer nuclear membrane, we showed an intriguing substrate specificity of several zDHHCs towards closely related substrates. Moreover, our data indicate that the steady-state localization of Gao at the PM and Golgi apparatus is the outcome of local S-palmitoylation events. These findings contrast the traditional views that i) Spalmitoylation of PMPs occurs exclusively at the Golgi and ii) serves to drive subsequent PM-directed delivery of such proteins. The unexpected selectivity among different PATs and their substrates we uncover to drive the intracellular localization of PMPs emerges as an attractive target for drug discovery. 


\section{RESULTS}

\section{The minimal localization code in the N-terminus of Gao}

For its membrane association, the $\mathrm{N}$-terminus of Gao is lipid modified by $\mathrm{N}$-myristoylation at Gly2 and S-palmitoylation at Cys $3^{7}$. A recent structural analysis identified the recognition sequence of N-Myristoyltransferases (NMTs) as an N-terminal hexapeptide, excluding Met $1^{16}$. This suggest that a minimal membrane-binding information might reside within the first seven residues of Gao. Thus far, three crystal structures of heterotrimeric Go have been solved ${ }^{17-19}$, showing a prominent $\alpha$-helix in the Gao N-terminus that extends toward the GßY heterodimer. An overlay of the $\mathrm{N}$-terminal region of these three structures revealed a similar ending of the $\alpha$-helixes, whereas their beginning falls on different residues at positions between 6 to 10 (Supplementary Fig. 1a-c). A similar overlay of the $\mathrm{N}$-terminal region of seven solved structures of Gai1 - a close Gao homologue - showed a much more consistent beginning for the $\alpha$-helix, which starts at residues 7 or 8 (Supplementary Fig. 1d-f). To study if these regions might have specific roles in Gao membrane association, we generated the following GFP-fusion constructs (Fig. 1a): one including Gao N-terminal heptapeptide (Gao$\mathrm{Nt}^{7}$-GFP), another with the first 31 residues covering the $\alpha$-helix (Gao-Nt ${ }^{31}-\mathrm{GFP}$ ), and the third containing only the $\alpha$-helix (Gao-Nt8-31-GFP). These constructs were expressed in the mouse neuroblastoma Neuro-2a cells (N2a) and their localization at the plasma membrane (PM) and Golgi apparatus were compared with the full-length Gao-GFP6 ${ }^{6}$. Surprisingly, Gao$\mathrm{Nt}^{7}$-GFP localized predominantly at the Golgi, whereas Gao-Nt ${ }^{31}$-GFP displayed a more homogenous PM and Golgi distribution similar to Gao-GFP (Fig. 1b). Quantification of average fluorescence intensities at these compartments confirmed a much higher Golgi and a lower PM localization of Gao-Nt ${ }^{7}$-GFP compared to Gao-Nt ${ }^{31}$-GFP and Gao-GFP (Fig. $1 \mathrm{c}, \mathrm{d})$. Then, we performed a crude subcellular fractionation of $\mathrm{N} 2 \mathrm{a}$ cells expressing the constructs and showed that Gao-Nt ${ }^{7}-\mathrm{GFP}, \mathrm{Gao}-\mathrm{Nt}^{31}$-GFP and Gao-GFP were similar in the way they partitioned between the cytosolic and membrane fractions (Fig. 1e,f). As opposed to these constructs showing prominent membrane localizations, Gao-Nt8-31-GFP was spread over the cytosol and nucleus (Supplementary Fig. 1g), indicating that the N-terminal $\alpha$-helix alone is not sufficient for membrane association.

In the heterotrimeric $\mathrm{G}$ protein complex, the $\mathrm{N}$-terminal $\alpha$-helix of $\mathrm{G} \alpha$ is one of the regions binding to $G \beta^{20,21}$. Since $G \beta$ tightly interacts with $G y$, which in turn associates to membranes via its C-terminal prenylation ${ }^{22}$, we tested if the poor PM localization of Gao-Nt ${ }^{7}-G F P$ relates to a lack of $G \beta y$ interaction. For this aim, we co-expressed the Gao constructs together with mRFP-G 31 and mRFP-Gy3 in N2a cells, and immunoprecipitated the GFP-fusions. Full-

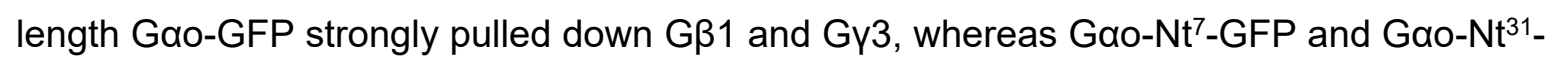


GFP showed a very faint co-precipitation of G $\beta y$ with no apparent difference between them (Supplementary Fig. $1 \mathrm{~h}$ ). Thus, the preferential localization of Gao-Nt-GFP to the Golgi, as opposed to the dual localization of Gao-Nt ${ }^{31}$-GFP to Golgi and PM, is independent from G $\beta$.

Together, these results indicate that $\mathrm{Nt}^{7}$ is sufficient for Gao overall membrane binding, but it seems to drive the Golgi rather than the PM localization.

\section{Key residues in Gao-Nt ${ }^{7}$}

We next aimed to decode the intracellular localization of Gao-Nt ${ }^{7}$ by a systematical point mutation analysis of its key residues. Amino acid substitutions were designed using the GPSLipid prediction tool ${ }^{23}$. For instance, we introduced the Gly-to-Leu mutation at position 2 of Gao-Nt ${ }^{7}$-GFP as GPS-Lipid predicted very high likelihood for S-palmitoylation at Cys3 in the mutant (not shown). However, the Gly2 mutation led to a complete loss of membrane association as Gao-G2L-Nt7 -GFP evenly spread over the cytosol and nucleus in N2a cells (Fig. 1g). This result reinforces the notion that $\mathrm{N}$-myristoylation is a prerequisite for $\mathrm{S}$ palmitoylation in $\mathrm{Gao}^{7}$. Consequently, Gao-G2L-Nt'-GFP appeared almost exclusively at the cytosolic fraction (Fig. 1h,i). We then introduced the Cys-to-Asn mutation at position 3 as GPS-Lipid predicted a high score for N-myristoylation at Gly2 in the mutant (not shown). The resulting Gao-C3N-Nt ${ }^{7}-$ GFP was predominantly excluded from the nucleus and showed an ER-like distribution with certain accumulation at the Golgi, which might be accounted for by the high membrane density of the cisternae stack (Fig. 1g). Nevertheless, Gao-C3N-Nt ${ }^{7}-G F P$ appeared mostly at the cytosolic fraction (Fig. 1h,i), confirming that $\mathrm{N}$-myristoylation confers only weak membrane binding properties ${ }^{8}$. Importantly, the localization of Gao-Nt ${ }^{7}$ mutants impaired in N-myristoylation and S-palmitoylation was emulated by the treatment with the specific inhibitors DDD85646 24 and 2-bromopalmitate (2-BrPal) ${ }^{25}$, respectively (Fig. 1j).

The most recent substrate recognition motif for NMTs was described as $\mathrm{M}^{1} \mathrm{G}\left[{ }^{\wedge} \mathrm{DEFRWY}\right] \mathrm{X}\left[{ }^{\wedge} \mathrm{DEKR}\right][\mathrm{ACGST}][\mathrm{KR}]^{7}$, with ${ }^{\wedge}$ denoting exclusion of residues, and $\mathrm{X}-$ any amino acid $^{16}$ The authors did not exclude the possibility that peptides lacking a positive charge at position 7 might also be accepted by NMTs. Thus, N-myristoylation of Gao - and all human N-myristoylated Ga subunits - is not in conflict with the lack of a Lys/Arg residue at this position (Fig. 1a). At position 6, a Ser residue is found in Gao and all its homologues, and Ser is by far the most frequent residue at this position among NMT substrates ${ }^{16}$. Thus, we first introduced point mutations at Ser6 in Gao- $\mathrm{Nt}^{7}$ based on the substrate recognition motif described by Castrec et al. and on a previous study indicating that Arg, Asn, Phe, and Val can occupy this position as well ${ }^{26}$. Substitution of Ser6 by Ala, Cys, Gly, or Thr displayed efficient membrane binding in N2a cells (Fig. 1k). Interestingly, the S6C mutant showed a much higher PM localization compared to Gao-Nt-GFP (Fig. 1k,I), suggesting that Cys3 and 
Cys6 both undergo S-palmitoylation and that PM targeting might be enhanced by the dual Spalmitoylation. $\mathrm{N}$-myristoylation of these mutants was indirectly verified with the inhibitor DDD85646 (Fig. 1k). Conversely, we observed a largely cytosolic and nuclear localization for the constructs in which the Ser6 was substituted by Arg, Asn, Phe and Val (Supplementary Fig. 1i), suggesting that these residues in position 6 make the N-terminal peptide of Gao a poor substrate for MNTs.

\section{Cysteine position}

Intrigued by the S6C mutant, we questioned if the position of the Cys residue within $\mathrm{Gao}^{-\mathrm{Nt}^{7}}$ might play any role in its subcellular localization. Thus, we moved the Cys 3 to positions 4 (MGNC-Nt ${ }^{7}$ ) and 5 (MGNTC-Nt ${ }^{7}$ ), and expressed the GFP constructs in N2a cells. An Asp residue was placed at position 3 following the GPS-Lipid prediction tool (not sown). Unexpectedly, MGNC-Nt ${ }^{7}-G F P$ displayed a completely different localization than its parental Gao-Nt ${ }^{7}$ (Figs. 1b, 2a). It exhibited PM localization that was not only higher than that of Gao$\mathrm{Nt}^{7}$-GFP, but also of the full-length Gao-GFP (Figs. 1b, 2b). Moreover, the perinuclear structures labelled by MGNC-Nt7-GFP showed a much lower co-localization with the Golgi marker GM130 compared to Gao-Nt-GFP (Pearson's correlation; Figs. 1b, 2a,c). Instead, the perinuclear structures containing MGNC-Nt ${ }^{7}-$ GFP co-localized better with Lactadherin-C2 (mRFP-Lact-C2; Supplementary Fig. 1j,k), a biosensor for phosphatidylserine ${ }^{27}$. These results imply that MGNC-Nt ${ }^{7}$-GFP preferentially associates with the PM-derived endocytic rather than the Golgi-derived secretory pathway. The distinct localization of MGNC-Nt'-GFP was not the result of a major change in the overall membrane binding, as its presence in the membrane fraction was not significantly different from that of Gao-Nt ${ }^{7}-$ GFP (Fig. 1h,i). SPalmitoylation of Cys4 in MGNC-Nt7-GFP was confirmed by the incubation with the inhibitor 2-BrPal (Fig. 2d). On the other hand, the Cys5 construct MGNTC-Nt ${ }^{7}-G F P$ localized at the Golgi and cytosol (Fig. 2a,c) but appeared mostly in the cytosolic fraction upon cell fractionation (Fig. 1h,i). Thus, it seems that the Cys5 is not a good substrate for palmitoyl acyltransferases (PATs), as MGNTC-Nt7 -GFP closely resembles the C3N mutant (Fig. 1g-i).

Most intriguingly, the subcellular localization of MGNC-Nt in $\mathrm{N} 2 \mathrm{a}$ cells was fully recapitulated in the full-length Gao when the same mutation was introduced. Specifically, MGNC-Gao-GFP showed a higher PM targeting (Figs. 1b, 2e,f) and a much lower co-localization with the Golgi marker GM130 (Figs. 1b, 2e,g) than Gao-GFP.

Altogether, these data uncover a remarkable characteristic of the $\mathrm{N}$-terminal localization code of Gao: minor changes in its amino acid sequence can drastically modify the subcellular localization of the protein. Due to its homology with all dual lipidated Ga subunits, and to 
other similarly modified PMPs, we reasoned that this principle might have a much broader relevance in biology.

\section{The evolution of $\mathbf{G a - N t ^ { 7 }}$}

Next, we wanted to determine how much diversity is present in $\mathrm{Nt}^{7}$ among eukaryotic $\mathrm{Ga}$ subunits. We searched for Ga sequences containing an N-terminal heptapeptide with the signature $M^{1} G X X X[A C G S T] X^{7}$, with at least one Cys at positions 3-5 or 7. Of note, we did not find any Ga subunit orthologue in the clades Ancyromonadida, Apusozoa, Breviatea, Cryptophyceae, CRuMs, Glaucocystophyceae, Malawimonadidae, and Rhodophyta (red algae). We found, however, Ga subunits in abundance in Amoebozoa, Discoba, Haptista, Metamonada, Opisthokonta, Sar, and Viridiplantae. Overall, we identified more than 800 sequences containing the desired $\mathrm{Nt}^{7}$ signature (Supplementary Table 1), and uncovered some interesting features. For instance, no Ga subunits was found with a Cys occupying position 7. On the other hand, we observed Cys throughout positions 3-5, with Cys 3 as the most common case with just over half of the sequences. Cys4 follows as the second most frequent with one third of all cases, whereas only $\sim 6 \%$ of Ga subunits contain a Cys 5 . The vast majority of $\mathrm{Ga}$ subunits containing a Cys5 are from plants ( 65\%) and Amoebozoa $(\sim 25 \%)$. Curiously, Cys5 is not present in Fungi and is almost completely absent in Metazoa, with the genus Caenorhabditis as the sole identified exception (e.g. C. elegans; Supplementary Table 1). Less than $10 \%$ of all sequences present two Cys residues occupying positions $3-5$, and they almost exclusively belong to fungal $\mathrm{Ga}$ subunits (Supplementary Table 1). A double Cys at positions 3 and 4 is by far the most frequent combination among them. No Ga sequence was found with a Cys occupying all three positions.

We asked whether consensus sequences might emerge from the Ga subunits among each category. As available databases are biased towards certain clades, we first selected unique hexapeptides with the signature $\mathrm{M}^{1} \mathrm{GXXX}[\mathrm{ACGST}]^{6}$. When an identical combination within positions 3-5 was found, we selected the sequence with the most frequent residue at position 7 for this analysis. Thus, we identified 88 unique combinations for Cys 3,75 for Cys 4 , and 13 for Cys5 (Supplementary Table 2). As seen in the corresponding Logo representations, there is no obvious Ga-Nt ${ }^{7}$ consensus for the Cys3 and Cys4 categories (Fig. 2h,i), whereas the sequence MGSLCSR emerges for Cys5 (Fig. 2j). Plotting the amino acids by their polarity groups showed a common characteristic among categories: positively and even more so negatively charged amino acids are strongly underrepresented at positions 3-5 compared to a random distribution, or even completely absent as in the Cys 5 category (Supplementary Fig. 2a-d). One can speculate that charged amino acids might interfere with at least one of 
the lipid modifications of $\mathrm{Ga}-\mathrm{Nt}^{7}$. The most recent recognition motif for NMTs (see above) denotes that positive and negative residues are not favored at certain positions ${ }^{16}$. Accordingly, N-myristoylation of Gao-Nt7-GFP appeared strongly impaired in N2a cells when different combinations of Arg, Asp, Glu, or Lys were introduced at positions 4 and 5 (Supplementary Fig. 2e). A Gao-Nt7 -GFP mutant with a His occupying position 5 showed a near normal membrane association regardless of the presence of a positive or negative residue at position 4 (Supplementary Fig. 2f). Thus, N-myristoylation of $\mathrm{Ga}^{-\mathrm{Nt}^{7}}$ of the Cys3 category appears optimal when Arg, Asp, Glu, or Lys residues are excluded from position 5, in total agreement with the substrate recognition motif described by Castrec et al.

As a consensus emerged for the Cys5 category (Fig. 2j), we expressed a GFP-fusion of this sequence in N2a cells. Somehow predictably, MGSLCSR-Nt-GFP showed a much better membrane binging than our designed MGNTC-Nt ${ }^{7}-$ GFP, and localized predominantly at the Golgi (Fig. 2a,k). When MGSLCSR was introduced into the full-length Gao, however, the membrane association of this mutant was not very efficient (Fig. 2l), probably related to the fact that Ga subunits of the Cys5 category are virtually absent in Metazoa (Supplementary Table 1).

Then, we expressed our constructs Gao-Nt7 -GFP, MGNTC-Nt7-GFP and the consensus MGSLCSR-Nt ${ }^{7}$-GFP in the Drosophila Schneider-2 cell line. Remarkably, all constructs retained the same localization patterns as seen in N2a cells: Gao-Nt ${ }^{7}$ and MGSLCSR-Nt ${ }^{7}$ targeted mainly Golgi stacks labelled by GalT-mRFP, while MGNTC-Nt ${ }^{7}$ associated largely with the PM (Fig. 2m). Similar localizations were also seen in HeLa cells (Supplementary Fig. $2 \mathrm{~g}$ ). These data point to highly conserved rules for $\mathrm{N}$-myristoylation and S-palmitoylation as well as for substrate compartmentalization.

\section{Direct targeting of $\mathrm{Nt}^{7}$ variants to Golgi or PM}

Our next goal was to understand the molecular mechanism behind the contrasting subcellular localization of two rather similar sequences: Gao-Nt ${ }^{7}$ and MGNC-Nt ${ }^{7} . \mathrm{N}-$ myristoylation generally occurs during protein synthesis and confers a weak membrane binding to the modified protein ${ }^{25}$. N-myristoylation is essential for the subsequent Spalmitoylation to occur ${ }^{7}$. Our own data showed that myristoylated, but not palmitoylated Gao$\mathrm{Nt}^{7}$ and MGNC-Nt ${ }^{7}$ are indistinguishable in their localization and only prevented from free cytosolic and nuclear diffusion (Figs. 1j, 2d). Therefore, we speculated that S-palmitoylation is key for subcellular compartmentalization, by itself or in combination with the myristoyl group and/or neighboring amino acids. It has been shown that S-palmitoylation of PMPs including Ga subunits - occurs exclusively at the Golgi apparatus, the event followed by the transport of PMPs to the PM via the secretory pathway ${ }^{13}$. Thus, one plausible explanation for 
the distinct steady-state localizations of Gao-Nt ${ }^{7}$ and MGNC-Nt ${ }^{7}$ might involve a differential Golgi-to-PM transport of the constructs.

To test this idea, our first approach was to visualize how these constructs are trafficked in the cell. We adapted to our constructs the reverse dimerization (RD) assay, originally designed for the synchronized trafficking of secretory and integral membrane proteins ${ }^{28}$. This method is based on the aggregation of the F36M mutant of the protein FKBP12 (FM) that allows protein tracking upon chemical dissociation. Thus, we intercalated four $\mathrm{FM}$ copies $\left(\mathrm{FM}^{4}\right)$ between $\mathrm{Nt}^{7}$ and GFP, and expressed the constructs in HeLa cells for better visualization. The distinct localization of Gao-Nt ${ }^{7}$ and MGNC-Nt ${ }^{7}$ - still observed in HeLa cells (Supplementary Fig. $2 \mathrm{~g}$ ) - was lost by the FM ${ }^{4}$ insertion, and cytosolic aggregates of different sizes were visualized instead (Fig. 3a). After chemical dissociation by the D/D solubilizer, most of the aggregates vanished and the constructs showed their characteristic localizations (Fig. 3a).

Then, live imaging was performed in HeLa cells co-expressing each of the $\mathrm{Nt}^{7}-\mathrm{FM}^{4}-\mathrm{GFP}$ constructs together with Mannll-mRFP, a broader Golgi marker. As expected, Gao-Nt7-FM ${ }^{4}-$ GFP showed a rapid accumulation at the Golgi once the D/D solubilizer was added (Fig. 3b,c and Supplementary Video 1). On the other hand, MGNC-Nt7 ${ }^{7} \mathrm{FM}^{4}-$ GFP presented a rather slow and miniscule accumulation at the Golgi, whereas its PM targeting was much faster (Fig. 3b,c and Supplementary Video 2). This result does not support a Golgi-to-PM flow of MGNC-Nt ${ }^{7}-\mathrm{FM}^{4}-\mathrm{GFP}$, as PM targeting was not preceded by its Golgi accumulation. To further prove that PM localization of MGNC-Nt ${ }^{7}-\mathrm{FM}^{4}-\mathrm{GFP}$ is indeed independent from transport through the Golgi, we performed the RD assay under the $20^{\circ} \mathrm{C}$ temperature block that forces cargo to accumulate in the Golgi ${ }^{29}$. As control, we used the GFP-FM4-hGH construct that aggregates in the ER lumen and is secreted via the Golgi-mediated transport after chemical dissociation ${ }^{30}$. Incubation with $D / D$ solubilizer for 45 min induced an almost complete secretion of GFP-FM ${ }^{4}-\mathrm{hGH}$ at $37^{\circ} \mathrm{C}$, while at $20^{\circ} \mathrm{C}$ the construct strongly accumulated in the Golgi (Fig. 3d). Conversely, HeLa cells expressing MGNC-Nt-FM4-GFP showed a comparable PM localization of the construct at both temperatures (Fig. 3d). Quantification of MGNC-Nt ${ }^{7}-\mathrm{FM}^{4}-\mathrm{GFP}$ mean fluorescence intensity in the Mannll-mRFP region revealed no significant difference in its Golgi content at $37^{\circ} \mathrm{C}$ and $20^{\circ} \mathrm{C}$ (Fig. 3e), supporting the notion that PM targeting of MGNC-Nt ${ }^{7}$ does not occur via transport through Golgi.

Altogether, these results imply that the characteristic steady-state localizations of $\mathrm{Gao}^{-\mathrm{Nt}^{7}}$ and MGNC-Nt ${ }^{7}$ are not related to the Golgi-to-PM trafficking. Instead, each of the constructs goes directly to its primary destination: Golgi or PM. 


\section{Lipid binding of $\mathrm{Nt}^{7}$ variants does not explain the preferential localization}

Thus far, our data indicate that S-palmitoylation might contribute to the compartmentalization of Gao-Nt ${ }^{7}$ and MGNC-Nt ${ }^{7}$, and that intracellular trafficking is not a major player in their distinctive localizations. We then envisioned two further scenarios that could explain the differential $\mathrm{Nt}^{7}$ compartmentalization: (i) PATs have no specificity for substrate recognition, and the substrates concentrate at different compartments due to specific interactions, and (ii) PATs discriminate substrates, which in turn accumulate at the compartment where Spalmitoylation takes place. The first scenario involves a certain degree of promiscuity among PATs ${ }^{12}$, and implies that lipidations and a few surrounding amino acids confer specific binding properties e.g. to lipids, which are known to differentially accumulate all over the endomembrane system ${ }^{31}$. Additionally, fatty acids other than palmitate can also be attached to the available Cys and might confer different binding specificities to the S-acylated protein $^{32}$. The second scenario requires that PATs discriminate between analogous substrates as small as $\mathrm{Nt}^{7}$ and that a given substrate accumulates at the compartment where its specific PAT is localized.

To test the first scenario, we used commercially available membrane strips spotted with fifteen different lipids found in cellular membranes and performed a protein-lipid overlay assay $^{33}$. As control, we employed a GFP-fusion of the pleckstrin homology $(\mathrm{PH})$ domain of FAPP1 (FAPP1-PH-GFP), that specifically binds to phosphatidylinositol 4-phosphate $(\mathrm{PI} 14 \mathrm{P})^{34}$. N2a cells were transfected with Gao-Nt7-GFP, MGNC-Nt ${ }^{7}-$ GFP or FAPP1-PH-GFP, and then membrane strips were incubated with cleared cell extracts. As expected, FAPP1PH-GFP showed a strong binding to the spot containing PI4P (Supplementary Fig. 3a). On the other hand, no apparent difference was found for Gao-Nt ${ }^{7}-\mathrm{GFP}$ and MGNC-Nt ${ }^{7}-\mathrm{GFP}$ that mainly bound to phosphatidylserine, cardiolipin, and phosphoinositides, particularly to PI4,5P2 (Supplementary Fig. 3a). A similar lipid-binding pattern was also observed by the larger construct Gao-Nt ${ }^{31}$-GFP (Supplementary Fig. 3a). Thus, the compartmentalization of Gao-Nt ${ }^{7}$ and MGNC-Nt ${ }^{7}$ is unlikely to emerge from different lipid-binding affinities, arguing against the first scenario.

\section{A novel tool for visualization of local S-palmitoylation in intact cells}

Next, we studied the second scenario, which implies that local S-palmitoylation drives substrate compartmentalization. In mammals, intracellular S-palmitoylation is mediated by 23-24 zDHHC proteins with PAT activity ${ }^{11}$. Most of the zDHHCs have four transmembrane domains (TMDs), whereas only two and three members present five and six TMDs, respectively ${ }^{12}$. The majority of $z D H H C s$ localize at the Golgi and the remaining are distributed among the ER, PM and endosomes ${ }^{35,36}$. In order to confirm their subcellular localization, we 
expressed a collection of twenty-three HA-tagged mouse zDHHCs in N2a cells ${ }^{37}$. As expected, immunostainings revealed that a large number of ZDHHCs localized predominantly at the Golgi: zDHHC3, 7, 9, 12, 13, 15-17, 21, 23, and 25 (Supplementary Fig. 3b). Six showed a clear PM localization but were also found on endosomes: zDHHC2, 5, 11, 14, 18, and to a lesser extent zDHHC8. The rest of the PATs mostly showed an ER localization, while zDHHC4 associated almost exclusively with the nuclear envelope (Supplementary Fig. 3b), a pattern not described in previous studies ${ }^{35,36}$.

Beside from a unique antibody that specifically recognizes palmitoylated PSD-95 ${ }^{38}$, experimental approaches to study zDHHC-substrate pair relations are based exclusively on disruptive biochemical and proteomic techniques ${ }^{39,40}$. To determine if Gao-Nt ${ }^{7}$ and MGNC$\mathrm{Nt}^{7}$ are differently lipidated at the PM vs. Golgi, we aimed at developing a method to visualize local S-palmitoylation in intact cells. Inspired by the nuclear envelope localization of zDHHC4, we engineered zDHHCs that ectopically target the outer nuclear membrane (ONM) to detect mislocalized substrates. We took advantage of some components of the wellstudied LINC (linker of nucleoskeleton and cytoskeleton) complexes. LINC complexes are built by proteins of the KASH (Klarsicht, ANC-1, and Syne Homology) family that are embedded in the ONM and interact within the perinuclear space with proteins of the SUN (Sad1 and UNC-84) family, which in turn are inserted in the inner nuclear membrane ${ }^{41}$. As the C-terminus of all zDHHCs except zDHHC24 faces the cytosol, we hypothesized that addition of a C-terminal KASH-domain might induce their targeting to the ONM (Fig. 4a). To test this hypothesis, we first generated a zDHHC5 construct carrying an N-terminal mRFP for visualization and the $\mathrm{KASH}$-domain of syne-1/nesprin-1 at its C-terminus (mRFP-zDHHC5KASH; Fig. 4a). In HeLa cells, the KASH-domain alone (mRFP-KASH) is efficiently targeted to the ONM (Fig. 4b), and mRFP-zDHHC5 localized at the PM and endosomes as expected (Fig. 4b and Supplementary Fig. 3b). The addition of the KASH domain strongly impaired PM localization of zDHHC5, but a robust ONM targeting was only achieved by the co-expression of the KASH-interacting protein SUN2 (Fig. 4b).

Next, we analyzed if known zDHHC-substrate pair relations can be recapitulated in HeLa cells and chose the PMPs SNAP23, caveolin-1 and flotillin-2. The core of SNAP23 contains 6 Cys residues that are plamitoylated by $z \mathrm{DHHC} 13$ and $17^{42}$. $\mathrm{ZDHHC} 7$ appears as the main PAT for caveolin-1, which is plamitoylated in 3 Cys residues at its C-terminus ${ }^{43}$. Flotillin-2 is myristoylated at Gly2 and palmitoylated at Cys 4,19 and 20 by zDHHC $5^{44}$. Remarkably, the GFP-fusions of SNAP23 (Supplementary Fig. 4a), caveolin-1 (Supplementary Fig. 4b) and flotillin-2 (Supplementary Fig. 4c) were efficiently recruited to the ONM by the corresponding zDHHC-KASH constructs. Together, we successfully developed a system in intact cells for the visualization of local S-palmitoylation at the ONM (henceforth SpONM). 


\section{Compartment-specific S-palmitoylation of $\mathrm{Nt}^{7}$}

Once the SpONM system was established, we first tested if PM-associated zDHHCs showed differential substrate specificities for Gao-Nt ${ }^{7}$ and MGNC-Nt ${ }^{7}$. In addition to zDHHC5, the Cterminal KASH-domain effectively targeted zDHHC2, 8, 11, 14 and 18 to the ONM (Fig. 4c,d and Supplementary Fig. 4d). Remarkably, Gao-Nt ${ }^{7}$-GFP and MGNC-Nt ${ }^{7}-$ GFP were efficiently recruited to the ONM by the co-expression of mRFP-zDHHC11-KASH but not by the control mRFP-KASH (Fig. 4c,d). To quantify these effects, we measured the mean GFP-

fluorescence intensity at the ONM and nearby cytosol, and used their ratio to determine the relative ONM content of the $\mathrm{Nt}^{7}$ constructs. Quantification revealed a similar $\sim 2.5$-fold accumulation of Gao-Nt ${ }^{7}-$ GFP and MGNC-Nt ${ }^{7}$-GFP at the ONM by the co-expression of mRFP-zDHHC11-KASH (Fig. 4e), suggesting that zDHHC11 equally accepts both $\mathrm{Nt}^{7}$ constructs as substrate and identifying this enzyme as the major PM-localized PAT for Gao. On the other hand, the KASH-fusions of $\mathrm{ZDHHC2}, 5,8,14$ and 18 showed no effect on the localization of Gao-Nt - GFP and MGNC-Nt ${ }^{7}$-GFP (Supplementary Fig. 4d). Thus, it appears

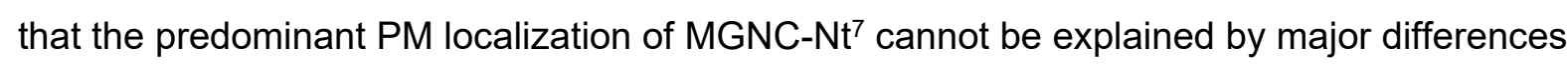
in substrate specificity of PM-associated zDHHCs.

Next, we applied the SpONM system to Gao-Nt ${ }^{7}$ and MGNC-Nt ${ }^{7}$ using the eleven Golgiassociated zDHHCs, as all were efficiently targeted to the ONM by the C-terminal KASHdomain (Fig. 4f,g and Supplementary Fig. 5a,b). Notably, the mRFP-zDHHC-KASH constructs for zDHHC3 and 7 caused a strong ONM accumulation of Gao-Nt-GFP (Fig. $4 \mathrm{f}, \mathrm{g}$ ). No other zDHHCs showed any activity toward Gao-Nt-GFP (Supplementary Fig. 5a), suggesting a high degree of specificity in substrate recognition among Golgi-associated PATs and identifying zDHHC3 and 7 as the major Golgi-localized PATs for Gao. MGNC-NtGFP was, however, only slightly recruited to the ONM by the KASH-fusions of zDHHC3 and 7 (Fig. 4f,g), and completely absent from the ONM by the co-expression of the remaining Golgi zDHHC-KASH constructs (Supplementary Fig. 5b). Quantification showed that Gao$\mathrm{Nt}^{7}$-GFP accumulates at the ONM roughly 2.0 and 2.5 folds by the co-expression of zDHHC3 and zDHHC7, respectively (Fig. 4h,i). Conversely, MGNC-Nt ${ }^{7}-$ GFP presented a significantly lower ONM recruitment of $\sim 1.5$ folds by both zDHHC3 and 7 (Fig. 4h,i).

To confirm that the PAT activity was responsible for the mislocalization of substrates in the SpONM system, we generated zDHHC-inactive constructs by a Cys-to-Ser mutation in the catalytic DHHC domain ${ }^{37}$. As anticipated, the ability of the KASH-fusions of zDHHC3, 7 and 11 to induce ONM accumulation of Gao-Nt ${ }^{7}$ and MGNC-Nt ${ }^{7}$ was completely abolished when DHHS-mutants were co-expressed (Fig. 5a). Together, our data suggest that the distinct steady-state localizations of Gao-Nt ${ }^{7}$ and $\mathrm{MGNC}-\mathrm{Nt}^{7}$ are due to the differential substrate 
specificities of PM- and Golgi-localized zDHHCs. This notion implies that the localization of $\mathrm{Nt}^{7}$ constructs might be shifted toward the PM or Golgi by manipulating the expression level of specific zDHHCs. To test this hypothesis, we co-transfected N2a cells with Gao-Nt ${ }^{7}-\mathrm{GFP}$ and HA-zDHHC11, and determined its relative PM content and co-localization with the MannII-BFP Golgi marker. We also co-expressed MGNC-Nt'-GFP together with HA-zDHHC3 or HA-zDHHC7. Remarkably, the PM localization of Gao-Nt ${ }^{7}$ strongly increased by the overexpression of zDHHC11, whereas its co-localization with Mannll-BFP showed a significant decrease (Fig. 5b,e,f). On the other hand, MGNC-Nt ${ }^{7}$ presented a higher colocalization with the Golgi marker but a significant decrease in PM content by the coexpression of zDHHC3 or zDHHC7 (Fig. 5c-f). The effects of the different zDHHCs on the steady-state localization of Gao-Nt ${ }^{7}$ and MGNC-Nt ${ }^{7}$ were not evident upon co-expression of the corresponding DHHS-mutants (Fig. 5b-f), since both $\mathrm{Nt}^{7}$ constructs showed similar localization patterns as described above (Figs. 1b, 2a-c). Collectively, these data confirm the notion that local S-palmitoylation plays a major role in the compartmentalization of $\mathrm{Gao}^{-\mathrm{Nt}^{7}}$ and MGNC-Nt ${ }^{7}$. As the KASH-fusion of $\mathrm{ZDHHC} 11$ was able to target both Gao-Nt ${ }^{7}$ and MGNC-Nt ${ }^{7}$ to the ONM (Fig. 4c-e), we asked whether it might not discriminate among different $\mathrm{Nt}^{7}$ substrates. We challenged this notion and found an interesting selectivity for closely related $\mathrm{Nt}^{7}$ sequences: mRFP-zDHHC11-KASH induced a strong ONM recruitment of MGSSCSR-Nt ${ }^{7}-G F P$ but showed a much weaker effect on MGLLCSR-Nt ${ }^{7}-G F P$ (Supplementary Fig. 6a,b), both sequences commonly found in plant Ga subunits (Supplementary Table 1). Thus, it appears that zDHHC3, 7 and 11 can differentiate among $\mathrm{Nt}^{7}$ substrates with slight variations in amino acid composition.

We then asked whether the higher PM targeting of Gao-Nt ${ }^{31}$ compared to Gao- $\mathrm{Nt}^{7}$ might also be the outcome of different zDHHC substrate specificities. Thus, we tested if zDHHC3, 7 , and 11 target Gao-Nt ${ }^{31}$ to the ONM with the same efficiency than Gao-Nt ${ }^{7}$. Although the three zDHHC-KASH constructs relocalized Gao-Nt ${ }^{31}$-GFP to the ONM, zDHHC3 and zDHHC7 showed a weaker effect when compared to Gao-Nt7 -GFP (Fig. 6a,b). Therefore, the weaker activity of the Golgi zDHHC3 and 7 toward Gao-Nt ${ }^{31}$ predicts an increase in its PM accumulation upon time.

Finally, we analyzed if the SpONM system might reveal local S-plamitoylation of the fulllength Gao. Thus, we expressed Gao-GFP together with the KASH-fusions of zDHHC3, 7, and 11 , as they showed activity toward Gao-Nt². Remarkably, full-length Gao-GFP was targeted to the ONM by all zDHHC constructs (Fig. 6c). On the other hand, the Cys4 mutant of Gao, MGNC-Gao-GFP, localized at the ONM primarily when the KASH-fusion for zDHHC11 was co-expressed (Supplementary Fig. 6c), similar to what we observed for MGNC-Nt ${ }^{7}$-GFP (Fig. 4d,f,g). Overall, our SpONM system shows that local S-palmitoylation 
of Gao can cause its in-situ accumulation, indicating that zDHHC selectivity and subcellular localization are key players in the substrate compartmentalization. 


\section{DISCUSSION}

That $\mathrm{G} \alpha$ subunits can be found at the Golgi apparatus is known for almost 30 years ${ }^{45}$. The Golgi localization of Ga subunits was assumed to be part of their trafficking pathway to the cell periphery, although some non-canonical functions of G-proteins were described at compartments other than the $\mathrm{PM}^{46}$. Only recently, the Golgi-pool of $\mathrm{G} \alpha$ subunits has been implicated in the downstream signaling of typical GPCRs ${ }^{4}$ and the KDEL receptor ${ }^{5,6}$. Thus, subcellular compartmentalization has emerged as an important player in $\mathrm{G}$ protein functions. The present study provides an in-depth characterization of key elements controlling $\mathrm{N}$ myristoylation, S-palmitoylation and localization of Gao. More importantly, we developed a system - SpONM - for the rapid detection of $\mathrm{ZDHHC}$-substrate pair relations in intact cells, opposing the disruptive biochemical and proteomic techniques currently used to study Spalmitoylation ${ }^{39}, 40$. Altogether, this work supports a model in which local S-palmitoylation by distinct zDHHCs is crucial for Gao compartmentalization, and most likely for other PMPs (Fig. 6d). In this model, N-myristoylation allows the nascent protein to reach virtually the whole endomembrane system. Subsequently, specific PM- and Golgi-localized zDHHCs catalyze S-palmitoylation of PMPs, which in turn accumulate at these compartments. Our model is in agreement with the local S-palmitoylation described for substrates such as PSD95 at dendritic spines ${ }^{38}$ and calnexin at the $\mathrm{ER}^{47}$, but adds the Golgi as a compartment in which PMPs can be locally modified and retained. To our knowledge, S-palmitoylation via the large number of Golgi-localized zDHHCs has been exclusively discussed in the context of Golgi-to-PM transport of integral ${ }^{48}$ and $\mathrm{PMPs}^{13}$. We hereby challenge the current model and show instead that PMP localization to one compartment or another (including Golgi and $\mathrm{PM}$ ) is not a consequence of sequential trafficking of the PMPs but rather a direct localization driven by the substrate-specific PAT activities at different compartments.

The fact that zDHHCs can modify multiple substrates has led to the notion that Spalmitoylation is nonspecific and proximity-based. Accordingly, a recent study demonstrated the stochastic S-palmitoylation of engineered Cys in membrane proteins, proposing that catalysis is determined by substrate accessibility and not specific sequence motifs ${ }^{49}$. Several zDHHCs, however, contain PDZ-binding motifs, SH3 domains or ankyrin-repeats outside the catalytic DHHC core that have been implicated in enzyme-substrate interactions ${ }^{12}$. Importantly, our study uncovered a striking substrate specificity for several zDHHCs toward peptides as small as Gao-Nt². We additionally showed that minor sequence modifications might result not only in drastic changes in the substrate recognition by zDHHCs, but also in the substrate localization (Fig. 6d). Another interesting implication of this model is that cells and tissues could shift the localization - and associated function - of PMPs toward one specific compartment or another by controlling the expression of $\mathrm{zDHHCs}$. The large number 
of $z \mathrm{DHHC}$ genes and their distinct tissue-specific expression patterns in humans is thus not surprising ${ }^{35}$. This property might be widespread among Metazoa as $\mathrm{Nt}^{7}$ constructs showed remarkably similar localization in Drosophila and mammalian cells, and Drosophila contains also a large number of $z D H H C$ genes that are differentially expressed in embryonic and adult tissues ${ }^{50}$. Moreover, it is tempting to speculate that clade-specific $\mathrm{ZDHHC}$ specializations might account for the distinct Cys position in eukaryotic Ga subunits, especially for Cys at position 5 that dominates in plants and amoebae but is almost absent in Metazoa.

The SpONM system we developed in this study relies on tagging zDHHCs at their Cterminus with the KASH domain of syne-1/nesprin-1 for the ectopic ONM targeting. We applied this strategy only to PM- and Golgi-localized zDHHCs but it might also work for the remaining PATs, excluding zDHHC24 with a predicted C-terminus facing the lumen ${ }^{40}$. Some of the ER-localized zDHHCs, however, showed a prominent ONM localization that might hinder their usage in the SpONM system. In this case, alternative tags for the targeting of ER-localized zDHHCs to the PM, Golgi or mitochondria might be applicable. The C-terminal domain of zDHHCs contains two non-catalytic TTXE and PaCCT (palmitoyltransferase conserved C-terminus) motifs that seem relevant for the overall protein structure ${ }^{51}$. The extreme C-terminal region of zDHHCs appears unstructured and is most likely not involved in the binding and presentation of substrates ${ }^{12}$, implying that the fusion of a $\mathrm{KASH}$ domain does not interfere with the substrate specificity. Accordingly, our SpONM pointed to zDHHC3, 7, and 11 as the major PATs for Gao. This result is in agreement with a previous study showing zDHHC3 and 7 as the main PATs for Gai2, Gaq and Gas ${ }^{52}$, although zDHHC11 activity toward $\mathrm{Ga}$ subunits has not been detected. This discrepancy could be explained by a differential turnover rate of PM- and Golgi-localized Ga subunits. For instance, if the turnover of the PM pool of Gai2 is much faster than its Golgi pool, the S-palmitoylation of Gai2 by the PM-localized zDHHC11 might have gone unnoticed by the biochemical methods used in that study. The SpONM system might therefore provide an advantage in sensitivity by targeting zDHHCs to the ONM, as even small amounts of a substrate are visible at the ectopic compartment.

A polybasic stretch present in the $\mathrm{N}$-terminal region of all $\mathrm{Ga}$ subunits has been implicated in $\mathrm{PM}$ binding ${ }^{53}$. The Gao-Nt ${ }^{31}$ construct containing the basic region within the $\alpha$-helix showed indeed a greater steady-state localization at the PM compared to Gao-Nt². In the SpONM system, the presence of the $\alpha$-helix in Gao-Nt ${ }^{31}$ appears to impact substrate recognition by the Golgi-associated zDHHC3 and 7, but not by the PM-localized zDHHC11. Thus, these data point out to a second level of complexity in substrate affinity by zDHHCs, where regions distant from the palmitoylatable Cys might subserve regulatory functions. How the polybasic region in Gao impacts substrate specificity by zDHHCs remains to be clarified. 
S-palmitoylation has gained much attention lately as a potential target for drug discovery to combat pathologies mediated by proteins such as the oncogenic Ras and infectious diseases like malaria caused by Plasmodium falciparum or the respiratory syndromes caused by coronaviruses $^{54-56}$. Our SpONM system could enable screens for molecules that perturb siteand zDHHC-specific S-palmitoylation of pathological proteins. This approach may provide an attractive alternative to directly target the desired oncogene (or protein of another pathogenic activity), focusing instead on developing tools to target the zDHHCs controlling the proper (or pathological) localization of the protein. In this regard, zDHHCs can be targeted by the drug discovery efforts. The large number of these enzymes in humans and their distinct expression patterns provide the reasoning to expect the power of such an approach in obtaining the favorable pharmacodynamics with limited side effects. Another approach might aim at targeting the specific zDHHC-substrate interaction pairs; this avenue is expected to deliver even more specific targeted therapies.

To sum up, we have delineated the code describing the Golgi vs. PM localization of the G protein Gao, showed that these two localizations are independent from the traditionally assumed Golgi-to-PM trafficking nor preferential interactions with major lipids, and discovered a universal mechanism controlling PMP localization to its target membrane through substrate selectivity of differently localized PATs. This major insight into the cell biology of protein subcellular localization may pave the way to novel drug discovery principles. 


\section{METHODS}

\section{Antibodies and reagents}

Monoclonal antibodies (mAbs) against flotillin-2 (Cat\# 610383) and GM130 (Cat\# 610823) were from BD Biosciences, mAb against HA (Cat\# 11867423001) from Roche, mAb against mRFP (Cat\# sc-101526) from Santa Cruz Biotechnology, mAb against GAPDH (Cat\# GTX28245) and polyclonal antibody (pAb) against GFP (Cat\# GTX113617) were from GeneTex. Secondary Abs for immunostaining and Western blots were from Jackson ImmunoResearch. DAPI (Cat\# 32670) and Cycloheximide (Cat\# C4859) were from SigmaAldrich, D/D solubilizer (Cat\# 635054) from Clontech, VECTASHIELD Mounting Medium (Cat\# H-1400) from Vector Laboratories, Glutathione Sepharose beads (Cat\# GE17-075601) from GE Healthcare, DDD85646 (Cat\# 13839) from Cayman Chemical, 2bromopalmitate (Cat\# sc-251714) from Santa Cruz Biotechnology, and Membrane lipid strips (Cat\# P-6002) from Echelon Biosciences.

\section{$\underline{\text { Cell lines and culture conditions }}$}

Male mouse neuroblastoma Neuro-2a (N2a; ATCC CCL-131) and female human epithelial HeLa (ATCC CCL-2) cells were maintained in MEM (Thermo Fisher Scientific), supplemented with 10\% FCS, $2 \mathrm{mM} \mathrm{L-glutamine,} 1 \mathrm{mM}$ pyruvate, and $1 \%$ penicillinstreptomycin at $37^{\circ} \mathrm{C}$ and $5 \% \mathrm{CO}_{2}$.

Male Drosophila Schneider-2 (S2) cells were maintained in Schneider's Drosophila Medium (Lonza) supplemented with $10 \% \mathrm{FCS}$ and $1 \%$ penicillin-streptomycin at $28^{\circ} \mathrm{C}$.

All vector transfections were carried out with X-tremeGENE 9 or HP (Roche) according to manufacturer's instructions.

\section{Plasmids and molecular cloning}

The plasmids Gao-GFP, GalT-GFP, and GalT-mRFP for Drosophila expression were previously described ${ }^{6}$. To generate the GFP-fusion of Gao-Nt ${ }^{7}$ and Gao-Nt ${ }^{31}$, the fragments were PCR-amplified (primers listed in Supplementary Table 3) from Gao-GFP, products were cut with $\mathrm{Kpnl} / \mathrm{Agel}$ and cloned in frame into the same sites of pEGFP-N1 (Clontech). The Gao-Nt8-31-GFP sequence was PCR-amplified from Gao-Nt ${ }^{31}$-GFP, the product cut with $\mathrm{Kpnl} / \mathrm{Notl}$ and used to replace the GFP sequence in pEGFP-N1 cut with the same enzymes. Gao-Nt ${ }^{7}$-GFP was used as template for the PCR amplification of the following $\mathrm{Nt}^{7}$-GFP sequences: Gao-G2L, Gao-C3N, Gao-S6A, Gao-S6C, Gao-S6G, Gao-S6T, Gao-S6F, GaoS6N, Gao-S6R, Gao-S6V, MGNC, MGNTC, MGCKR, MGCKE, MGCDR, MGCDE, MGCKH, MGCDH, MGSLCSR, MGSSCSR, and MGLLCSR. All PCR products were cut with Kpnl/Notl and then used to exchange the corresponding GFP sequence from pEGFP-N1. For 
Drosophila expression, the plasmids containing the constructs Gao-Nt-GFP, MGNC-NtGFP, and MGSLCSR-Nt ${ }^{7}$-GFP were cut with EcoRI/Notl and inserts were ligated into the same sites of pAc5.1/V5-HisA (Thermo Fisher Scientific). The full-length Gao mutants MGNC-Gao and MGSLCSR-Gao were PCR-amplified from Gao-GFP, cut and inserted in frame into the Kpnl/Apal sites of pEGFP-N1. For the generation of Gao-Nt7-FM4-GFP and MGNC-Nt ${ }^{7}-\mathrm{FM}^{4}$-GFP, the FM ${ }^{4}$ sequence was PCR-amplified from the GFP-FM4-hGH plasmid (Andrew Peden, University of Sheffield), cut with Agel/Pstl and ligated in frame into the same sites of Gao-Nt ${ }^{7}-$ GFP and MGNC-Nt ${ }^{7}-$ FM $^{4}$-GFP. The mRFP-G $\beta 1$ construct was created by exchanging the Agel/BsrGI mCerulean sequence from mCerulean-G $\beta 1^{57}$ with the corresponding from pmRFP-C1 (Claudia Stuermer, University of Konstanz). Similarly, the mRFP-Gy3 was created by substituting the Agel/BsrGI GFP sequence in the GFP-Gy3 plasmid $^{6}$ with the mRFP sequence. The mRFP-Lact-C2 was done by replacing the Agel/BsrGI GFP sequence from GFP-Lact-C2 (kindly provided by Gregory Fairn; University of Toronto) with the sequence from pmRFP-C1. MannII-mRFP was created by exchanging the BamHI/Notl sequence from MannII-BFP ${ }^{58}$ with the equivalent sequence from pmRFP-N1 (Claudia Stuermer, University of Konstanz). The mRFP-KASH plasmid was cloned by substituting the Agel/BsrGI GFP sequence in the GFP-KASH plasmid ${ }^{59}$ with the analogous sequence from pmRFP-C1. The plasmid for the non-tagged SUN2 expression was cloned by cutting the SUN2-CFP plasmid ${ }^{60}$ with EcoRI and BamHI, blunting with Phusion DNA Polymerase (New England Biolabs), and re-ligating the plasmid thus introducing a stop codon in frame with the SUN2 sequence. The mRFP-zDHHC5 construct was cloned by cutting the BamHI/Notl sequence of $\mathrm{pCl}$-neo-Flag-DHHC5 ${ }^{61}$ and ligating in frame into the Bglll/PspOMI sites of pmRFP-C1. For the generation of mRFP-zDHHC5-KASH, the sequence mRFP-zDHHC5 was PCR-amplified from mRFP-zDHHC5, cut with Nhel/Kpnl and used to exchange the GFP sequence cut with the same enzymes from GFP-KASH. Masaki Fukata (National Institutes of Natural Sciences, Japan) generously provided a collection of 23 mouse $z \mathrm{DHHC}$ isoforms cloned into the pEF-Bos-HA plasmid ${ }^{37}$. Slightly different cloning strategies were used for the generation of all mRFP-zDHHC-KASH constructs. Specifically, the $z D H H C 2,11$ and 18 sequences were PCR-amplified from the corresponding pEF-Bos$\mathrm{HA}$ plasmids, cut with Agel/Kpnl and ligated in frame into the BspEl/Kpnl sites of mRFP$\mathrm{KASH}$. The sequence for $\mathrm{ZDHHC3}, 7,12,13,15,16,21$, and 25 were PCR-amplified from the original pEF-Bos-HA plasmids, cut with Bglll/EcoRI and inserted into the same sites of mRFP-KASH. The zDHHC8 sequence was PCR-amplified from pEF-Bos-HA-DHHC8, cut with Xhol/EcoRI and ligated into the same sites of mRFP-KASH. zDHHC9 was PCRamplified from pEF-Bos-HA-DHHC9, cut with Sacl/BamHI and ligated into the same sites of mRFP-KASH. The zDHHC14 sequence was PCR-amplified from pEF-Bos-HA-DHHC14, cut with Agel/BamHI and ligated into the BspEl/BamHI sites of mRFP-KASH. The zDHHC17 and 
23 were PCR-amplified from the corresponding pEF-Bos-HA plasmids, cut with BgllI/HindIII and inserted into the same sites of mRFP-KASH. All inactive DHHS-mutants for the relevant mRFP-zDHHC-KASH and HA-zDHHC constructs were obtained by point mutagenesis. The GFP-fusion of reggie-1/flotillin-2 was previously described ${ }^{62}$. Tamas Balla (National Institutes of Health) kindly provided the FAPP1-PH-GFP plasmid and Bo van Deurs (University of Copenhagen) the GFP-caveolin-1 construct. The GFP-SNAP2363 and pGEX6P1-GFPNanobody ${ }^{64}$ plasmids were obtained from Addgene

\section{$\underline{\text { Structure alignment }}$}

The following Gao structures (PDB IDs) $6 \mathrm{~g} 79^{17}, 6 \mathrm{oik}^{18}$, and $6 \mathrm{k} 41^{19}$ were aligned using the PyMOL software (pymol.org). The N-terminal $\alpha$-helix of $6 \mathrm{~g} 79$ was set as reference to align all structures. A similar alignment was done for the Gai1 structures $1 \mathrm{gg}^{20}, 5 \mathrm{kdo}^{65}, 6 \mathrm{ddf} 66$, $6 \mathrm{osa}{ }^{67}, 6 \mathrm{n} 4 \mathrm{~b}^{68}, 6 \mathrm{kpf}^{69}$, and $6 \mathrm{k} 42^{19}$, setting the $\mathrm{N}$-terminal $\alpha$-helix of $5 \mathrm{kdo}$ as reference.

\section{Immunofluorescence and microscopy}

For microscopy, N2a and HeLa cells were transfected for $7 \mathrm{hr}$, trypsinized and seeded on poly-L-lysine-coated coverslips in complete MEM for additional $15 \mathrm{hr}$ before fixation. When indicated, cells were seeded in complete MEM supplemented with $10 \mu \mathrm{M}$ DDD85646 or 100 $\mu \mathrm{M}$ 2-bromopalmitate. S2 cells were transfected for $24 \mathrm{hr}$, washed one time with PBS, resuspended in complete media, and seeded on poly-L-lysine-coated coverslips for 30 min before fixation. All cells were fixed for 20 min with $4 \%$ paraformaldehyde in PBS. For immunostaining, cells were permeabilized for 1 min using ice-cold PBS supplemented with $0.1 \%$ Triton $X-100$, blocked for 30 min with PBS supplemented with $1 \%$ BSA, incubated with the primary antibody in blocking buffer for $2 \mathrm{hr}$ at room temperature (RT), washed and subsequently incubated with secondary antibodies and DAPI in blocking buffer for $2 \mathrm{hr}$ at RT. All fluorescent-labelled secondary antibodies were from Jackson ImmunoResearch. Coverslips were finally mounted with Vectashield on microscope slides. Cells were recorded with a Plan-Apochromat 63x/1.4 oil objective on a LSM800 Confocal Microscope and further processed using the ZEN blue software (all Zeiss). When required (see below), mean fluorescence intensity was determined from confocal images using ImageJ (National Institutes of Health).

\section{$\underline{P M}$ and Golgi accumulation}

N2a cells expressing the GFP-fusion constructs were immunostained as indicated above using a mouse monoclonal antibody (mAb) against GM130 to visualize the Golgi apparatus, and a DAPI staining for the nucleus (both not shown in all final images). To avoid interferences due to different expression levels of GFP-constructs among cell population, mean fluorescence intensity was measured at the GM130-positive Golgi region as well as at 
the total cell area, and ratio values were used to determine the relative Golgi accumulation of the constructs. Simultaneously, mean fluorescence intensity was determined at an unbroken region of the PM lacking membrane protrusions, and the ratio over total cell fluorescence was used to define relative PM content for each GFP-construct. One-way ANOVA or Student's t-test were used for statistical analysis of $\geq 50$ cells per condition and from at least 3 independent experiments.

\section{Co-localization analysis}

N2a cells were transfected and immunostained against GM130 as described above. To determine co-localization of the various GFP constructs and the GM130 signal, an area covering the whole perinuclear region but excluding the PM was selected in confocal images and the Pearson's correlation was calculated using the co-localization tool of ImageJ. The same analysis was done in N2a cells co-expressing the phosphatidylserine biosensor mRFPLact-C2 ${ }^{27}$ and the different GFP-fusions, transfected at equal plasmid ratio. The Golgi marker GalT-GFP was used as control. This analysis was also done in N2a cells co-transfected with GFP-fusions, HA-tagged zDHHCs and Mannll-BFP as the Golgi marker (2:4:1 plasmid ratio). One-way ANOVA test was used for statistical analysis of $>30$ cells per condition.

\section{Co-immunoprecipitation}

The recombinant GST-tagged Nanobody against GFP ${ }^{64}$ expressed in Escherichia coli Rosettagami (Novagen) was purified with glutathione Sepharose 4B beads according to manufacturer's instructions. Protein purity was assessed by SDS-PAGE and Coomassie blue staining.

N2a cells were co-transfected with the different Gao-GFP constructs and mRFP-G $\beta 1 / G y 3$ at 1:1:1 plasmid ratio. After $24 \mathrm{hr}$ transfection, cells were resuspended with ice-cold GST-lysis buffer (1\% Triton X-100 and 10\% glycerol in PBS) supplemented with a protease inhibitor cocktail (Roche) and passed 10 times through a 25G needle. Extracts were cleared by centrifugation at $15,000 \mathrm{xg}$ for $20 \mathrm{~min}$ at $4^{\circ} \mathrm{C}$, and supernatants were incubated with $1 \mu \mathrm{g}$ of purified GST-tagged GFP-Nanobody for $30 \mathrm{~min}$ on ice. Then, $20 \mu \mathrm{L}$ of Glutathione Sepharose $4 \mathrm{~B}$ beads were added and samples rotated overnight at $4^{\circ} \mathrm{C}$. Beads were repeatedly washed with GST-lysis buffer, prepared for SDS-PAGE, and finally analyzed by Western blot using a rabbit polyclonal antibody (pAb) against GFP and a mouse mAb against mRFP. HRP-conjugated secondary pAbs (Jackson ImmunoResearch) were used for enhanced chemiluminescence $(E C L)$ detection. The co-immunoprecipitation was done in duplicate with very similar outcomes. 


\section{Crude subcellular fractionation}

N2a cells were transfected for $24 \mathrm{hr}$ and cell extracts were prepared using non-denaturing conditions and fractionated by high-speed centrifugation as previously described ${ }^{62}$. Briefly, cells were rinsed twice with PBS and resuspended in hypo-osmotic buffer (20 mM Hepes, pH 7.0) supplemented with a protease inhibitor cocktail. The cell suspension was passed 20 times through a $25 \mathrm{G}$ needle, and nuclei and unbroken cells were removed by centrifugation at $700 \mathrm{xg}$ for $10 \mathrm{~min}$ at $4^{\circ} \mathrm{C}$. The supernatant was centrifuged at $100,000 \mathrm{xg}$ for $60 \mathrm{~min}$ at $4^{\circ} \mathrm{C}$. The new supernatant (cytosolic fraction) was directly prepared for SDS-PAGE, and the pellet (membrane fraction) was gently washed with hypo-osmotic buffer and resuspended in GSTlysis buffer supplemented with $0.5 \%$ SDS and protease inhibitors. The membrane fraction was cleared by centrifugation at $15,000 \times \mathrm{xg}$ for $20 \mathrm{~min}$ at $4^{\circ} \mathrm{C}$, and prepared for SDS-PAGE. Western blot analysis was done as above using the pAb against GFP and the mouse mAbs against flotillin-2 and GAPDH as endogenous membrane and cytosolic markers, respectively. Two-way ANOVA was used for statistical analysis of 4-6 independent experiments.

\section{Protein-lipid overlay assay}

After $24 \mathrm{hr}$ of transfection, N2a cells were washed twice with TBS (50 mM Tris- $\mathrm{HCl}, \mathrm{pH}$ 8.0, $150 \mathrm{mM} \mathrm{NaCl}$ and $10 \%$ glycerol) and cell extracts were prepared as explained above in TBS supplemented with $0.5 \%$ Tx-100 and protease inhibitors. Membrane lipid strips were blocked in 3\% BSA in TBS supplemented with $0.1 \%$ Tween-20 (TBS-T) for $1 \mathrm{hr}$ at $4^{\circ} \mathrm{C}$. Then, strips were incubated overnight at $4^{\circ} \mathrm{C}$ with cleared cell extracts previously diluted $1 / 10$ in TBS-T supplemented with $1 \%$ BSA. Strips were incubated first with a pAb against GFP, and then with a secondary HRP-conjugated $\mathrm{pAb}$ to detect GFP-constructs bound to lipid dots. The protein-lipid overlay assay was done in duplicate for each condition with very similar results.

\section{Screening of eukaryotic Ga subunits}

To identify Ga subunit paralogs in eukaryotic genomes, the full-length amino acid sequence of Gao was used for search queries in the NCBI protein Blast browser

(blast.ncbi.nlm.nih.gov/Blast.cgi). Specifically, species with sequenced genomes were selected from Ensembl databases (ensembl.org) for vertebrates, plants, fungi, metazoa and protist. For the remaining phylogenetic clades, Ga paralogs were found as above and using the NCBI Taxonomy browser (ncbi.nlm.nih.gov/Taxonomy/Browser/wwwtax.cgi) as guide for clade classification. Sequences were manually curated and when annotated as partial were not considered further for this analysis. A total of 262 species were found to express $\mathrm{G} \alpha$ paralogs among the clades Amoebozoa, Discoba, Haptista, Metamonada, Opisthokonta, Sar, and Viridiplantae (Table S1). Logo representation of the unique sequences selected for the different Gao-Nt ${ }^{7}$ categories (Table S2) was done using the WebLogo application ${ }^{70}$ and further edited with Inkscape (inkscape.org). 


\section{Reverse dimerization assay}

HeLa cells were co-transfected with the Golgi marker GalT-mRFP (not shown in all figures) and Gao-Nt ${ }^{7}-F^{4}-$ GFP, MGNC-Nt ${ }^{7}-F^{4}$-GFP or GFP-FM+4-hGH (at a 1:3 plasmid ratio), and seeded on poly-L-lysine-coated coverslips for $15 \mathrm{hr}$ as indicated above. Then, cells were incubated for $2 \mathrm{hr}$ at normal culture conditions in MEM supplemented with $20 \mathrm{mM}$ Hepes, $\mathrm{pH}$ 7.4 and $50 \mu \mathrm{M}$ Cycloheximide to block de novo synthesis of proteins. The reverse dimerization was induced by adding fresh MEM supplemented as above plus $1 \mu M D / D$ solubilizer for the time indicated in the corresponding figures. For temperature block of Golgi transport, the last two steps were simultaneously performed at $37^{\circ} \mathrm{C}$ and $20^{\circ} \mathrm{C}$. Then, cells were fixed and prepared for microscopy. Quantification of fluorescence intensity at the Mannll-mRFP Golgi region was done as described above. Student's t test was used for statistical analysis of $\geq 40$ cells per condition and from 3 independent experiments.

For the live imaging of reverse dimerization, HeLa cells were transfected as above but seeded on $\mu$-Slide-4-wells coverslips (Ibidi). Cell were first incubated for $30 \mathrm{~min}$ at $37^{\circ} \mathrm{C}$ in Hank's Balanced Salt Solution (Gibco) supplemented with 20 mM Hepes, pH 7.4 and $50 \mu \mathrm{M}$ cyclohexamide. Slides were then mounted on a temperature-controlled stage in a VisiScope CSU-X1 spinning disk confocal system (Visitron Systems) equipped with a Plan-Apochromat 63x/1.4 oil objective on an AxioObserver.Z1 microscope (Zeiss), a Evolve 512 EMCCD Camera (Photometrics), and the VisiView Imaging software (Visitron Systems). Reverse dimerization was induced by adding $D / D$ solubilizer to reach a $1 \mu \mathrm{M}$ final concentration, and cells were immediately recorded at one image per 5 second for $10 \mathrm{~min}$. For analysis, movies were generated from stacks using ImageJ, and the mean fluorescence intensity of an area at the center of the Mannll-mRFP Golgi region was measured from stacks in >10 cells per condition.

\section{S-palmitoylation at the outer nuclear membrane (SpONM)}

HeLa cells were co-transfected as above with plasmids encoding the GFP-fusion, SUN2, and control mRFP-KASH or mRFP-zDHHC-KASH at a 1:3:3 plasmid ratio. Cells were then seeded on poly-L-lysine-coated coverslips, fixed and prepared for microscopy. For quantification, mean GFP-fluorescence intensity at the ONM region labelled by the mRFPKASH fusion was determined using ImageJ. Simultaneously, GFP-fluorescence was measured at a nearby cytosolic region as well, and the ratio ONM over cytosol was used to define the relative ONM content of each GFP-construct. One-way ANOVA or Student's t-test were used for statistical analysis of $\geq 40$ cells per condition and from at least 3 independent experiments. 


\section{Statistics and reproducibility}

Statistical parameters, including the exact values of $\mathrm{n}$ are reported in the figure legends.

Results are shown as the mean, and error bars represent the s.e.m. or s.d.; ns, not significant, ${ }^{*} p \leq 0.01 ;{ }^{* *} p \leq 0.005 ;{ }^{* * *} p \leq 0.001 ;{ }^{* * * *} p \leq 0.0001$ using one-way or two-way ANOVA with Tukey's post hoc test (for multi-sample groups), and two-sided unpaired Student's $t$-test or Mann-Whitney U test (for two-sample comparison). GraphPad Prism 7 was used to determine statistical significance. No statistical methods were used to predetermine the sample sizes. All replicates successfully reproduced the presented findings. 


\section{ACKNOWLEDGEMENTS}

The work was supported with the Swiss National Science Foundation grant 31003A_175658 to VLK.

\section{AUTHOR CONTRIBUTIONS}

G.P.S. performed and analyzed the experiments, and developed all methodologies. J.V. assisted with protein structural analysis. C.A. assisted with interpretation of results. G.P.S. and V.L.K. designed the work and wrote the paper.

\section{COMPETING INTERESTS}

The authors declare no competing interests. 


\section{REFERENCES}

1. Rosenbaum, D.M., Rasmussen, S.G. \& Kobilka, B.K. The structure and function of Gprotein-coupled receptors. Nature 459, 356-363 (2009).

2. Hauser, A.S., Attwood, M.M., Rask-Andersen, M., Schioth, H.B. \& Gloriam, D.E. Trends in GPCR drug discovery: new agents, targets and indications. Nat Rev Drug Discov 16, 829-842 (2017).

3. Oldham, W.M. \& Hamm, H.E. Heterotrimeric G protein activation by G-proteincoupled receptors. Nat Rev Mol Cell Biol 9, 60-71 (2008).

4. Lobingier, B.T. \& von Zastrow, M. When trafficking and signaling mix: How subcellular location shapes $\mathrm{G}$ protein-coupled receptor activation of heterotrimeric $\mathrm{G}$ proteins. Traffic 20, 130-136 (2019).

5. Luini, A. \& Parashuraman, S. Signaling at the Golgi: sensing and controlling the membrane fluxes. Curr Opin Cell Biol 39, 37-42 (2016).

6. Solis, G.P. et al. Golgi-Resident Galphao Promotes Protrusive Membrane Dynamics. Cell 170, 939-955 e924 (2017).

7. Chen, C.A. \& Manning, D.R. Regulation of $\mathrm{G}$ proteins by covalent modification. Oncogene 20, 1643-1652 (2001).

8. Farazi, T.A., Waksman, G. \& Gordon, J.I. The biology and enzymology of protein Nmyristoylation. J Biol Chem 276, $39501-39504$ (2001).

9. Linder, M.E. \& Deschenes, R.J. New insights into the mechanisms of protein palmitoylation. Biochemistry 42, 4311-4320 (2003).

10. Yuan, M. et al. N-myristoylation: from cell biology to translational medicine. Acta Pharmacol Sin (2020).

11. Gottlieb, C.D. \& Linder, M.E. Structure and function of DHHC protein Sacyltransferases. Biochem Soc Trans 45, 923-928 (2017).

12. Rana, M.S., Lee, C.J. \& Banerjee, A. The molecular mechanism of DHHC protein acyltransferases. Biochem Soc Trans 47, 157-167 (2019).

13. Rocks, O. et al. The palmitoylation machinery is a spatially organizing system for peripheral membrane proteins. Cell 141, 458-471 (2010).

14. Fukata, M., Sekiya, A., Murakami, T., Yokoi, N. \& Fukata, Y. Postsynaptic nanodomains generated by local palmitoylation cycles. Biochem Soc Trans 43, 199204 (2015).

15. Philippe, J.M. \& Jenkins, P.M. Spatial organization of palmitoyl acyl transferases governs substrate localization and function. Mol Membr Biol 35, 60-75 (2019).

16. Castrec, B. et al. Structural and genomic decoding of human and plant myristoylomes reveals a definitive recognition pattern. Nat Chem Biol 14, 671-679 (2018). 
17. Garcia-Nafria, J., Nehme, R., Edwards, P.C. \& Tate, C.G. Cryo-EM structure of the serotonin 5-HT1B receptor coupled to heterotrimeric Go. Nature 558, 620-623 (2018).

18. Maeda, S., Qu, Q., Robertson, M.J., Skiniotis, G. \& Kobilka, B.K. Structures of the M1 and M2 muscarinic acetylcholine receptor/G-protein complexes. Science $364,552-$ 557 (2019).

19. Yuan, D. et al. Activation of the alpha2B adrenoceptor by the sedative sympatholytic dexmedetomidine. Nat Chem Biol (2020).

20. Wall, M.A. et al. The structure of the G protein heterotrimer Gi alpha 1 beta 1 gamma 2. Cell 83, 1047-1058 (1995).

21. Lambright, D.G. et al. The 2.0 A crystal structure of a heterotrimeric G protein. Nature 379, 311-319 (1996).

22. Senarath, K. et al. Regulation of G Protein betagamma Signaling. Int Rev Cell Mol Biol 339, 133-191 (2018).

23. Xie, Y. et al. GPS-Lipid: a robust tool for the prediction of multiple lipid modification sites. Sci Rep 6, 28249 (2016).

24. Kallemeijn, W.W. et al. Validation and Invalidation of Chemical Probes for the Human N-myristoyltransferases. Cell Chem Biol 26, 892-900 e894 (2019).

25. Resh, M.D. Trafficking and signaling by fatty-acylated and prenylated proteins. Nat Chem Biol 2, 584-590 (2006).

26. Martinez, A. et al. Extent of N-terminal modifications in cytosolic proteins from eukaryotes. Proteomics 8, 2809-2831 (2008).

27. Yeung, T. et al. Membrane phosphatidylserine regulates surface charge and protein localization. Science 319, 210-213 (2008).

28. Rivera, V.M. et al. Regulation of protein secretion through controlled aggregation in the endoplasmic reticulum. Science 287, 826-830 (2000).

29. Matlin, K.S. \& Simons, K. Reduced temperature prevents transfer of a membrane glycoprotein to the cell surface but does not prevent terminal glycosylation. Cell $\mathbf{3 4}$, 233-243 (1983).

30. Gordon, D.E., Bond, L.M., Sahlender, D.A. \& Peden, A.A. A targeted siRNA screen to identify SNAREs required for constitutive secretion in mammalian cells. Traffic 11, 1191-1204 (2010).

31. Harayama, T. \& Riezman, H. Understanding the diversity of membrane lipid composition. Nat Rev Mol Cell Biol 19, 281-296 (2018).

32. Lemonidis, K. et al. Substrate selectivity in the zDHHC family of S-acyltransferases. Biochem Soc Trans 45, 751-758 (2017).

33. Dowler, S., Kular, G. \& Alessi, D.R. Protein lipid overlay assay. Sci STKE 2002, pl6 (2002). 
34. Balla, A., Tuymetova, G., Tsiomenko, A., Varnai, P. \& Balla, T. A plasma membrane pool of phosphatidylinositol 4-phosphate is generated by phosphatidylinositol 4kinase type-III alpha: studies with the $\mathrm{PH}$ domains of the oxysterol binding protein and FAPP1. Mol Biol Cell 16, 1282-1295 (2005).

35. Ohno, Y., Kihara, A., Sano, T. \& Igarashi, Y. Intracellular localization and tissuespecific distribution of human and yeast DHHC cysteine-rich domain-containing proteins. Biochim Biophys Acta 1761, 474-483 (2006).

36. Ernst, A.M. et al. S-Palmitoylation Sorts Membrane Cargo for Anterograde Transport in the Golgi. Dev Cell 47, 479-493 e477 (2018).

37. Fukata, M., Fukata, Y., Adesnik, H., Nicoll, R.A. \& Bredt, D.S. Identification of PSD-95 palmitoylating enzymes. Neuron 44, 987-996 (2004).

38. Fukata, Y. et al. Local palmitoylation cycles define activity-regulated postsynaptic subdomains. J Cell Biol 202, 145-161 (2013).

39. Peng, T., Thinon, E. \& Hang, H.C. Proteomic analysis of fatty-acylated proteins. Curr Opin Chem Biol 30, 77-86 (2016).

40. Zaballa, M.E. \& van der Goot, F.G. The molecular era of protein S-acylation: spotlight on structure, mechanisms, and dynamics. Crit Rev Biochem Mol Biol 53, 420-451 (2018).

41. Sosa, B.A., Kutay, U. \& Schwartz, T.U. Structural insights into LINC complexes. Curr Opin Struct Biol 23, 285-291 (2013).

42. Lemonidis, K., Sanchez-Perez, M.C. \& Chamberlain, L.H. Identification of a Novel Sequence Motif Recognized by the Ankyrin Repeat Domain of zDHHC17/13 SAcyltransferases. J Biol Chem 290, 21939-21950 (2015).

43. Tonn Eisinger, K.R. et al. Palmitoylation of caveolin-1 is regulated by the same DHHC acyltransferases that modify steroid hormone receptors. J Biol Chem 293, 1590115911 (2018).

44. Li, Y., Martin, B.R., Cravatt, B.F. \& Hofmann, S.L. DHHC5 protein palmitoylates flotillin-2 and is rapidly degraded on induction of neuronal differentiation in cultured cells. J Biol Chem 287, 523-530 (2012).

45. Stow, J.L. et al. A heterotrimeric $\mathrm{G}$ protein, $\mathrm{G}$ alpha i-3, on Golgi membranes regulates the secretion of a heparan sulfate proteoglycan in LLC-PK1 epithelial cells. J Cell Biol 114, 1113-1124 (1991).

46. Wedegaertner, P.B. G protein trafficking. Subcell Biochem 63, 193-223 (2012).

47. Lakkaraju, A.K. et al. Palmitoylated calnexin is a key component of the ribosometranslocon complex. EMBO J 31, 1823-1835 (2012).

48. Ernst, A.M., Toomre, D. \& Bogan, J.S. Acylation - A New Means to Control Traffic Through the Golgi. Front Cell Dev Biol 7, 109 (2019). 
49. Rodenburg, R.N.P. et al. Stochastic palmitoylation of accessible cysteines in membrane proteins revealed by native mass spectrometry. Nat Commun 8, 1280 (2017).

50. Bannan, B.A. et al. The Drosophila protein palmitoylome: characterizing palmitoylthioesterases and DHHC palmitoyl-transferases. Fly (Austin) 2, 198-214 (2008).

51. Rana, M.S. et al. Fatty acyl recognition and transfer by an integral membrane Sacyltransferase. Science 359 (2018).

52. Tsutsumi, R. et al. Identification of $\mathrm{G}$ protein alpha subunit-palmitoylating enzyme. Mol Cell Biol 29, 435-447 (2009).

53. Kosloff, M., Elia, N. \& Selinger, Z. Structural homology discloses a bifunctional structural motif at the N-termini of $\mathrm{G}$ alpha proteins. Biochemistry 41, 14518-14523 (2002).

54. Chen, B., Sun, Y., Niu, J., Jarugumilli, G.K. \& Wu, X. Protein Lipidation in Cell Signaling and Diseases: Function, Regulation, and Therapeutic Opportunities. Cell Chem Biol 25, 817-831 (2018).

55. Fung, T.S. \& Liu, D.X. Post-translational modifications of coronavirus proteins: roles and function. Future Virol 13, 405-430 (2018).

56. Ko, P.J. \& Dixon, S.J. Protein palmitoylation and cancer. EMBO Rep 19 (2018).

57. Thaler, C., Koushik, S.V., Blank, P.S. \& Vogel, S.S. Quantitative multiphoton spectral imaging and its use for measuring resonance energy transfer. Biophys $J$ 89, 27362749 (2005).

58. Subach, O.M., Cranfill, P.J., Davidson, M.W. \& Verkhusha, V.V. An enhanced monomeric blue fluorescent protein with the high chemical stability of the chromophore. PLoS One 6, e28674 (2011).

59. Espigat-Georger, A., Dyachuk, V., Chemin, C., Emorine, L. \& Merdes, A. Nuclear alignment in myotubes requires centrosome proteins recruited by nesprin-1. J Cell Sci 129, 4227-4237 (2016).

60. Turgay, Y. et al. SUN proteins facilitate the removal of membranes from chromatin during nuclear envelope breakdown. J Cell Biol 204, 1099-1109 (2014).

61. Tian, H. et al. Systematic siRNA Screen Unmasks NSCLC Growth Dependence by Palmitoyltransferase DHHC5. Mol Cancer Res 13, 784-794 (2015).

62. Solis, G.P. et al. Reggie/flotillin proteins are organized into stable tetramers in membrane microdomains. Biochem J 403, 313-322 (2007).

63. Kuster, A. et al. The Q-soluble N-Ethylmaleimide-sensitive Factor Attachment Protein Receptor (Q-SNARE) SNAP-47 Regulates Trafficking of Selected Vesicle-associated Membrane Proteins (VAMPs). J Biol Chem 290, 28056-28069 (2015). 
64. Katoh, Y., Nozaki, S., Hartanto, D., Miyano, R. \& Nakayama, K. Architectures of multisubunit complexes revealed by a visible immunoprecipitation assay using fluorescent fusion proteins. J Cell Sci 128, 2351-2362 (2015).

65. Kaya, A.I. et al. A Conserved Hydrophobic Core in Galphai1 Regulates G Protein Activation and Release from Activated Receptor. J Biol Chem 291, 19674-19686 (2016).

66. Koehl, A. et al. Structure of the micro-opioid receptor-Gi protein complex. Nature $\mathbf{5 5 8}$ 547-552 (2018).

67. Kato, H.E. et al. Conformational transitions of a neurotensin receptor 1-Gi1 complex. Nature 572, 80-85 (2019).

68. Krishna Kumar, K. et al. Structure of a Signaling Cannabinoid Receptor 1-G Protein Complex. Cell 176, 448-458 e412 (2019).

69. Hua, T. et al. Activation and Signaling Mechanism Revealed by Cannabinoid Receptor-Gi Complex Structures. Cell 180, 655-665 e618 (2020).

70. Crooks, G.E., Hon, G., Chandonia, J.M. \& Brenner, S.E. WebLogo: a sequence logo generator. Genome Res 14, 1188-1190 (2004). 


\section{FIGURE LEGENDS}

\section{Figure 1. Key amino acid residues in Gao N-terminus}

a, Amino acid sequence of the GFP-fusion constructs of Gao N-terminus. b-d,

Representative confocal images of N2a cells expressing full-length Gao-GFP (top), Gao-NtGFP (middle) or Gao-Nt ${ }^{31}$-GFP (bottom) and immunostained against GM130 as Golgi marker (b). Note that while Gao-Nt ${ }^{31}$-GFP localizes at the PM and Golgi similar to Gao-GFP, Gao$\mathrm{Nt}^{7}$-GFP targets mainly the Golgi. Color-channels are listed vertically top-to-bottom and selected areas are magnified with the channels displayed also vertically in the same order. DAPI staining in blue marks nuclei. Quantification of mean fluorescence intensity ratios of GFP-fusions at the Golgi (c) or PM (d) versus total cell. Data shown as the mean \pm s.e.m. from 4 independent experiments (Gao-Nt ${ }^{7}, n=56$; Gao-Nt ${ }^{31}, n=61$; Gao, $n=58$ ). e,f, Western blot of a crude subcellular fractionation of N2a cells expressing the constructs described in (b). Ab against GFP was used for detection of Gao constructs, and Abs against GAPDH and flotillin-2 (Flot-2) used as cytosolic (Cyto) and membrane (Mem) markers, respectively (e). Quantification of the relative distribution of GFP-fusions in cytosolic and membrane fractions (f). Data shown as the mean \pm s.e.m. from 6 independent experiments. g, Images of N2a cells expressing the mutants Gao-G2L-Nt7 -GFP (left) or Gao-C3N-Nt-GFP (right) and immunostained against GM130 (not showed in the left panel). Selected region is zoomed-in with DAPI staining in blue. $\mathbf{h}, \mathbf{i}$, Crude fractionation of N2a cells expressing Gao-Nt ${ }^{7}-\mathrm{GFP}$ or the different $\mathrm{Nt}^{7}$-GFP mutants indicated in the figure (h). Western blot analysis done as in (e). Relative distribution of GFP-fusions in the cytosolic and membrane fractions (i). Data shown as the mean \pm s.e.m. from 6 independent experiments. j, Localization of Gao-Nt ${ }^{7}-G F P$ in N2a cells under inhibition of $\mathrm{N}$-myristoylation (DDD85646) or S-palmitoylation (2-bromopalmitate; 2-BrPal). $\mathbf{k}, \mathrm{I}$, Localization in N2a cells of mutants of the Serine at position 6 (S6) in Gao-NtGFP under normal conditions (top) or inhibition of N-myristoylation (bottom). The Ser-to-Cys (S6C) mutant showed a much higher targeting to the PM, quantified in (I). Data shown as the mean \pm s.e.m. from 4 independent experiments (Gao-Nt ${ }^{7}, n=56$; S6C, $n=63$ ). b,g,j,k, scale bars, $10 \mu \mathrm{m}$. See also Supplementary Fig. 1.

\section{Figure 2. Position of the Cys in Gao N-terminus}

a-d, Representative confocal images of $\mathrm{N} 2 \mathrm{a}$ cells expressing the $\mathrm{Nt}^{7}$-GFP mutants MGNC (top) or MGNTC (bottom) and immunostained against GM130 (a). Note that MGNC-Nt-GFP localizes strongly at the PM and shows a poor co-localization with the Golgi marker, whereas MGNTC-Nt7'-GFP is mostly cytosolic and at the Golgi. Nuclei in blue stained with DAPI.

Selected areas are magnified to the right. Quantification of mean fluorescence intensity ratios 
of GFP-fusions at the PM versus total cell (b). Data shown as the mean \pm s.e.m. from 4 independent experiments (Gao-Nt ${ }^{7}, n=56$; MGNC-Nt ${ }^{7}, n=50$; Gao, $n=58$ ). Co-localization analysis of the $\mathrm{Nt}^{7}$-GFP constructs with GM130 (c). Data shown as the mean \pm s.e.m. from 3 independent experiments (Gao-Nt ${ }^{7}, n=33$; MGNC-Nt ${ }^{7}, n=36$; MGNTC-Nt ${ }^{7}, n=39$ ). Localization of MGNC-Nt-GFP under inhibition of S-palmitoylation (2-BrPal; d). e-g, Image of N2a cells expressing the MGNC mutant of full-length Gao (MGNC-Gao-GFP) and immunostained against GM130 (e). DAPI staining in blue. Boxed area is enlarged. Relative localization of GFP-fusions at the PM (f). Data shown as the mean \pm s.e.m. from 4 independent experiments (Gao, $n=56$; MGNC-Gao, $n=46$; MGNC-Nt ${ }^{7}, n=50$ ). Co-localization analysis of the constructs with GM130 $(\mathbf{g})$. Data shown as the mean \pm s.e.m. from 3 independent experiments (Gao, n=47; MGNC-Gao, n=38; MGNTC-Nt ${ }^{7}, n=36$ ). $\mathbf{h}$-j, Logo representations of unique eukaryotic $\mathrm{Ga}-\mathrm{Nt}^{7}$ sequences for the categories Cys3 (h), Cys4 (i) and Cys5 (j). $\mathbf{k}, \mathrm{I}, \mathrm{N} 2 \mathrm{a}$ cells expressing the $\mathrm{Nt}^{7}$-GFP construct with the consensus sequence for the Cys5 category (MGSLCSR; k). Marked region is magnified. Nucleus stained in blue with DAPI. Full-length Gao-GFP carrying the Cys 5 consensus $\mathrm{Nt}^{7}$ sequence expressed in N2a cells (I). m, Localization of Gao-Nt-GFP (top), MGNC-Nt ${ }^{7}-G F P$ (middle) and MGSLCSR-Nt7-GFP (bottom) in Drosophila S2 cells. Co-expression of GalT-mRFP labels Golgi stacks. Selected areas are zoomed-in to the right. a,e,d,k,I, scale bars, $10 \mu \mathrm{m} . \mathbf{m}$, scale bars, $5 \mu \mathrm{m}$. See also Supplementary Fig. 1 and 2, and Supplementary Tables 1 and 2.

\section{Figure 3. Targeting of $\mathbf{N t}^{7}$-GFP}

a, Reverse dimerization (RD) assay in HeLa expressing Gao-Nt7-FM+4-GFP (left panels) or MGNC-Nt ${ }^{7}-F^{4}{ }^{4}-G F P$ (right panels). Cytosolic clusters formed by the $\mathrm{Nt}^{7}-\mathrm{FM}^{4}$-GFP constructs (top) dissolve by the addition of D/D solubilizer (D/D-Sol, bottom) and show their expected localizations. b,c, Live imaging of HeLa cells co-expressing Gao-Nt-FM4-GFP (top) or MGNC-Nt ${ }^{7}-\mathrm{FM}^{4}$-GFP (bottom) and the Golgi marker Mannll-mRFP (bottom right insets). Representative images of the $\mathrm{Nt}^{7}-\mathrm{FM}^{4}$-GFP constructs at the time of D/D solubilizer addition $(0 \mathrm{~min})$ and after $6 \mathrm{~min}(\mathbf{b})$. Relative increase upon time of $\mathrm{Nt}^{7}-\mathrm{FM}^{4}$-GFP constructs at the Golgi region (c). Note that Gao-Nt7 -FM4-GFP quickly targets the Golgi region, while MGNC$\mathrm{Nt}^{7}-\mathrm{FM}^{4}$-GFP only slightly accumulates at the Golgi. Data represent mean \pm s.d. (Gao-Nt ${ }^{7}$, $n=12$; MGNC-Nt $\left.{ }^{7}, n=11\right)$. d,e, RD assay in HeLa cells expressing MGNC-Nt ${ }^{7}-F^{4}{ }^{4}-G F P$ (right) or the secretable control GFP-FM ${ }^{4}-\mathrm{hGH}$ (left) performed at $37^{\circ} \mathrm{C}$ or at $20^{\circ} \mathrm{C}$ to inhibit Golgi transport. Incubation with D/D solubilizer results in the almost complete secretion of GFP$\mathrm{FM}^{4}-\mathrm{hGH}$ at $37^{\circ} \mathrm{C}$ (middle left) but in a Golgi retention at $20^{\circ} \mathrm{C}$ (bottom left). PM targeting of MGNC-Nt ${ }^{7}-\mathrm{FM}^{4}$-GFP seems not affected by the $20^{\circ} \mathrm{C}$ temperature block (bottom right), and its presence at the Golgi region (Mannll-mRFP) is not higher than at $37^{\circ} \mathrm{C}$ (middle right). 
Quantification of mean fluorescence intensity of MGNC-Nt7-FM+4-GFP, Golgi over total cell (e). Data shown as mean \pm s.e.m. from 3 independent experiments $\left(37^{\circ} \mathrm{C}, n=49 ; 20^{\circ} \mathrm{C}\right.$, $n=40)$. a,b,d, scale bars, $10 \mu \mathrm{m}$. See also Supplementary Figure 2, and Supplementary Videos 1 and 2.

\section{Figure 4. Local S-palmitoylation at the ONM}

a, Schematic representation of core components of the S-palmitoylation at the ONM (SpONM) assay. b, Representative images of HeLa cells expressing the constructs mRFPKASH that targets the ONM, mRFP-zDHHC5 or mRFP-zDHHC5-KASH with or without the co-expression of SUN2 (not shown). Note that mRFP-zDHHC5-KASH efficiently targets the ONM in the presence of SUN2. c-e, The SpONM assay for Gao-Nt ${ }^{7}-G F P(\mathbf{c})$ and MGNC-Nt ${ }^{7}$ GFP (d) using the control mRFP-KASH constructs or mRFP-zDHHC11-KASH. Note that the expression of the KASH-construct carrying zDHHC11 results in the strong recruitment of Gao-Nt7 -GFP (bottom; c) and MGNC-Nt7-GFP (bottom; d) at the ONM, effect not seen by the control mRFP-KASH (top; c,d). Nuclei stained in blue with DAPI, and marked regions are magnified to the right. Quantification of mean fluorescence intensity ratios of GFP-fusions at the ONM versus cytosol (e). Data shown as the mean \pm s.e.m. from 3 independent

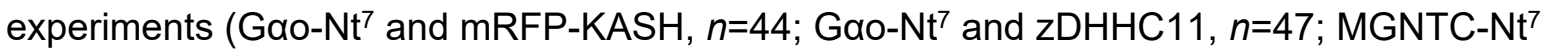
and mRFP-KASH, $n=50$; MGNTC-Nt ${ }^{7}$ and zDHHC11, $n=43$ ). $\mathbf{f - i}$, The SpONM assay using zDHHC3 (f) and zDHHC7 (g). Gao-Nt7-GFP efficiently targets the ONM by the co-expression of mRFP-zDHHC3-KASH (top; f) and mRFP-zDHHC7-KASH (top; $\mathbf{g}$ ) in HeLa cells. MGNC$\mathrm{Nt}^{7}$-GFP poorly localizes at the ONM in the presence of mRFP-zDHHC3-KASH (bottom; $\mathbf{f}$ ) and mRFP-zDHHC7-KASH (bottom; g). DAPI stained nuclei in blue and selected areas are magnified to the right. Quantification of mean fluorescence intensity ratios of GFP-fusions at the ONM versus cytosol by zDHHC3 (h) and zDHHC7 (i). Data shown as the mean \pm s.e.m. from 3 independent experiments (Gao-Nt ${ }^{7}$ and mRFP-KASH, $n=44$; Gao-Nt ${ }^{7}$ and $z D H H C 3$, $n=50$; Gao-Nt ${ }^{7}$ and zDHHC7, $n=46$; MGNTC-Nt ${ }^{7}$ and mRFP-KASH, $n=50$; MGNTC-Nt ${ }^{7}$ and zDHHC3, $n=53$; MGNTC-Nt ${ }^{7}$ and zDHHC7, $\left.n=45\right)$. b,c,d,f,g, scale bars, $10 \mu \mathrm{m}$. See also Supplementary Figures 3 and 4.

\section{Figure 5. zDHHC expression levels drive $\mathrm{Nt}^{7}$ compartmentalization}

a, Gao-Nt7-GFP displays its characteristic localization in the SpONM assay using the inactive DHHS mutants for zDHHC3 (zDHHS3, top left), zDHHC7 (zDHHS7, bottom left) and zDHHC11 (zDHHS11, top right) in HeLa cells. The zDHHS11 mutant shows no effect in the localization of MGNC-Nt7-GFP (bottom right). Boxed areas are zoomed-in to the right, and DAPI labeling nuclei in blue. b-f, Representative images of N2a cells co-expressing Gao-Nt- 
GFP (b) or MGNC-Nt-GFP (c,d) together with HA-zDHHC11 (top; b), HA-zDHHC3 (top; c) or HA-zDHHC7 (top; d). The corresponding DHHS inactive mutants (bottom; b-d) were used as controls. Golgi was labelled by the co-expression of Mannll-BFP and HA-tagged zDHHCs were immunostained against HA (b-d). Color-channels are listed vertically top-to-bottom and selected areas are magnified with the channels displayed horizontally in the same order leftto-right. Quantification of mean fluorescence intensity ratios of GFP-fusions at the PM versus total cell $(\mathbf{e})$. Data shown as the mean \pm s.e.m. from 3 independent experiments (zDHHC11, $n=61$; zDHHS11, $n=54$; zDHHC3, $n=53$; zDHHS3, $n=56$; zDHHC7, $n=54 ; \mathrm{zDHHS7,} n=52$ ). Co-localization analysis of $\mathrm{Nt}^{7}-\mathrm{GFP}$ constructs with Mannll-BFP (f). Data represent mean \pm s.e.m. from 3 independent experiments (zDHHC11, $n=53$; zDHHS11, $n=53$; zDHHC3, $n=53$; zDHHS3, $n=51$; zDHHC7, $n=52$; zDHHS7, $n=55)$. a,b,c,d, scale bars, $10 \mu \mathrm{m}$. See also Supplementary Figure 5.

\section{Figure 6. Targeting of full-length Gao to the ONM}

a,b, The SpONM assay using the control mRFP-KASH, zDHHC3, zDHHC7 and zDHHC11 (a). Gao-Nt ${ }^{31}$-GFP efficiently targets the ONM by the co-expression of mRFP-zDHHC11$\mathrm{KASH}$, whereas mRFP-zDHHC3-KASH and mRFP-zDHHC7-KASH induce only a weak ONM recruitment. Quantification of mean fluorescence intensity ratio (ONM versus cytosol) of Gao-Nt ${ }^{31}$-GFP compared to Gao-Nt ${ }^{7}$-GFP $(\mathbf{b})$. Data shown as the mean \pm s.e.m. from 3 independent experiments (For Gao-Nt7: mRFP-KASH, $n=44$; zDHHC3, $n=50$; zDHHC7, $n=46$; zDHHC11, $n=47$. For Gao-Nt ${ }^{31}$ : mRFP-KASH, $n=42 ;$ zDHHC3, $n=45 ; \mathrm{zDHHC7}, n=41$; zDHHC11, $n=48$ ). c, Full-length Gao-GFP associates with the ONM in the SpONM assay with $\mathrm{zDHHC3}, \mathrm{zDHHC7}$ and $\mathrm{zDHHC11}$, but not with the control mRFP-KASH. d, Model of how some peripheral membrane proteins might achieve specific subcellular compartmentalization by the interplay of the substrate selectivity of different zDHHCs. The model uses Gao-Nt ${ }^{7}-$ GFP and MGNC-Nt ${ }^{7}-G F P$ as examples. a,c, Nuclei stained in blue with DAPI, boxed regions are magnified to the right, and scale bars, $10 \mu \mathrm{m}$. See also Supplementary Figure 6. 
bioRxiv preprint doi: https://doi.org/10.1101/2020.08.25.266692; this version posted August 26, 2020. The copyright holder for this preprint

(which was not certified by peer review) is the author/funder, who has granted bioRxiv a license to display the preprint in perpetuity. It is made available under aCC-BY-NC-ND 4.0 International license.

a

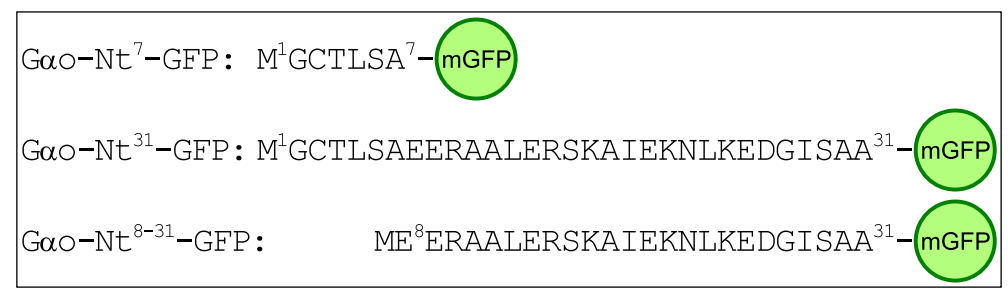

b

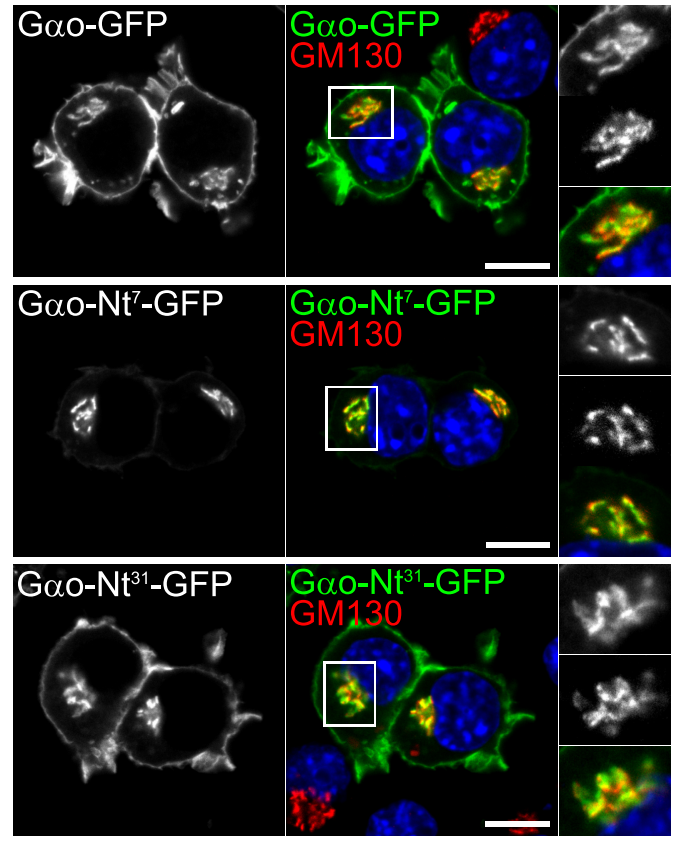

\section{g}
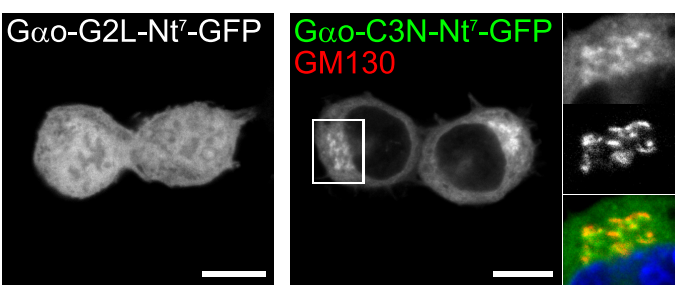

h

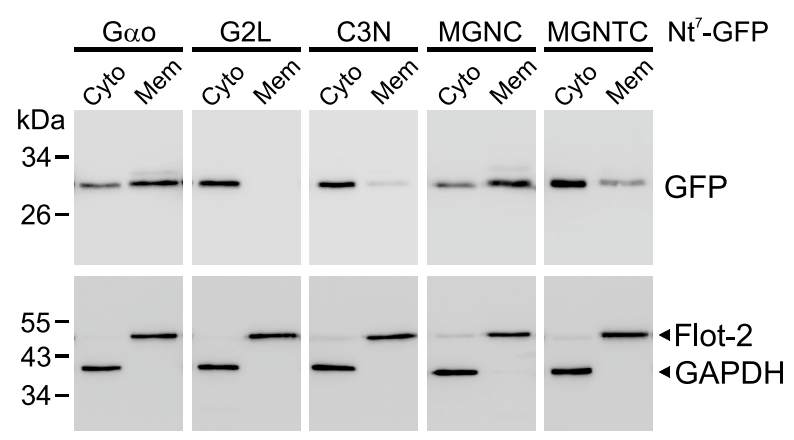

C
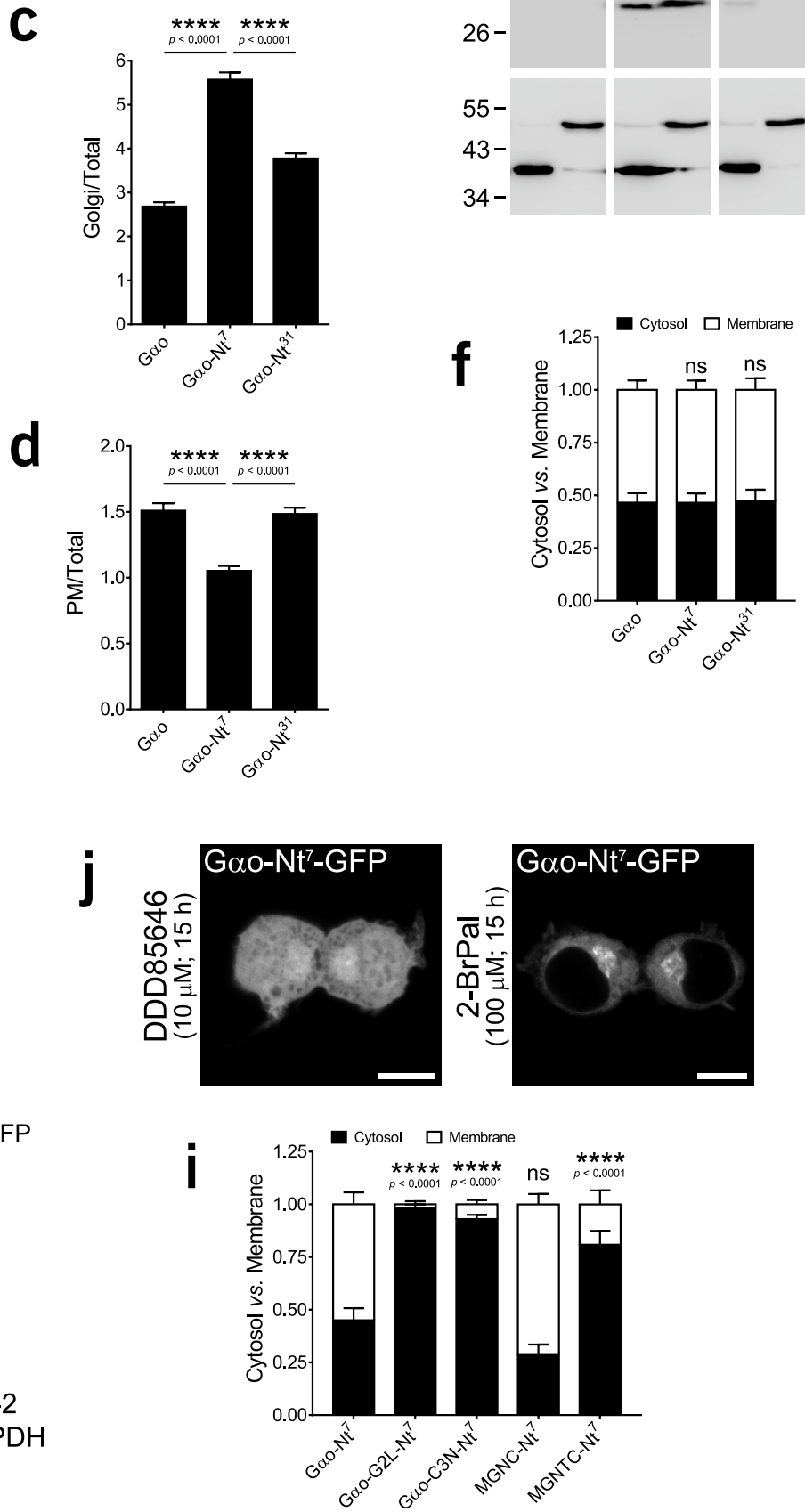
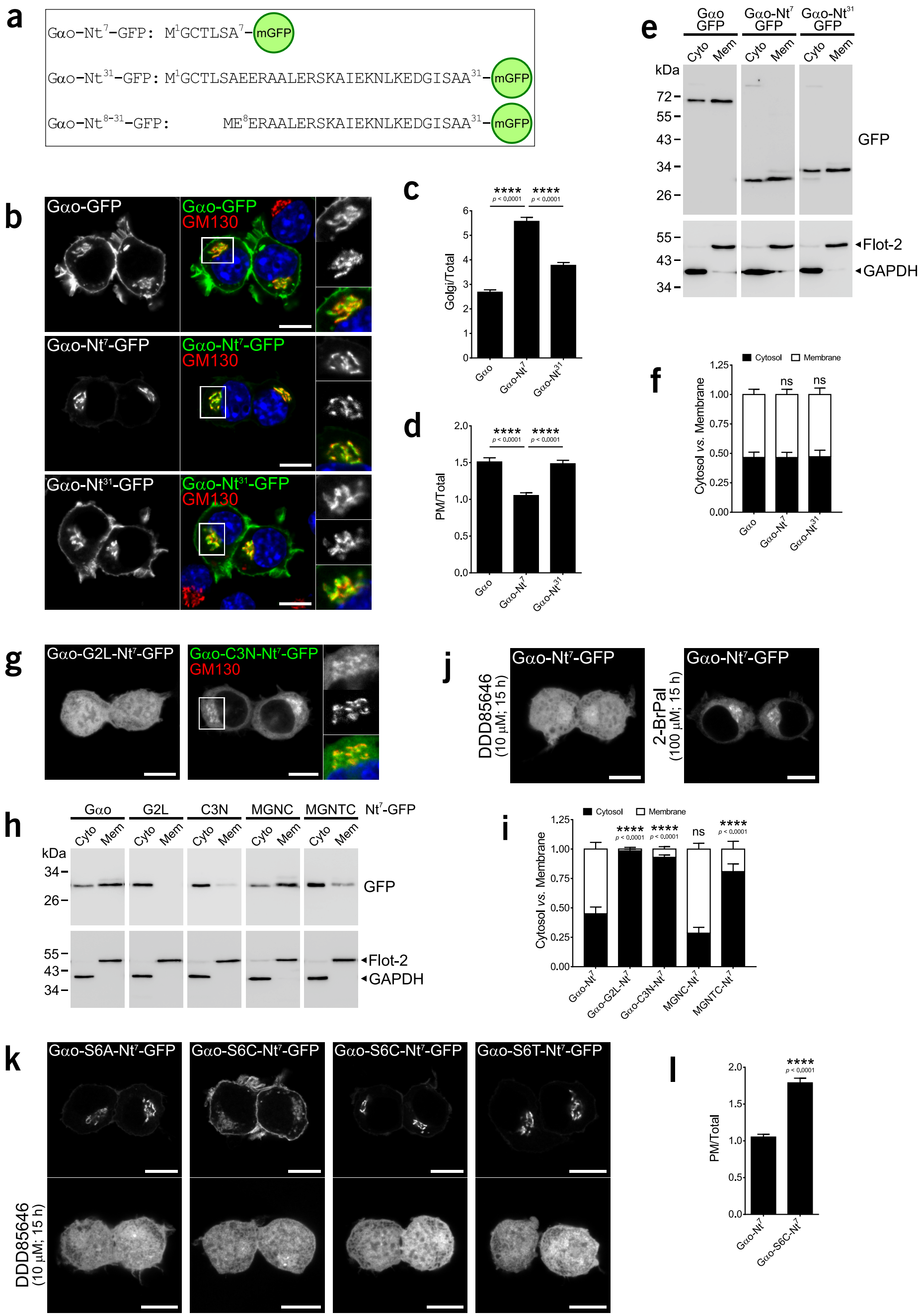
bioRxiv preprint doi: https://doi.org/10.1101/2020.08.25.266692; this version posted August 26, 2020. The copyright holder for this preprint

(which was not certified by peer review) is the author/funder, who has granted bioRxiv a license to display the preprint in perpetuity. It is made available under aCC-BY-NC-ND 4.0 International license.

a

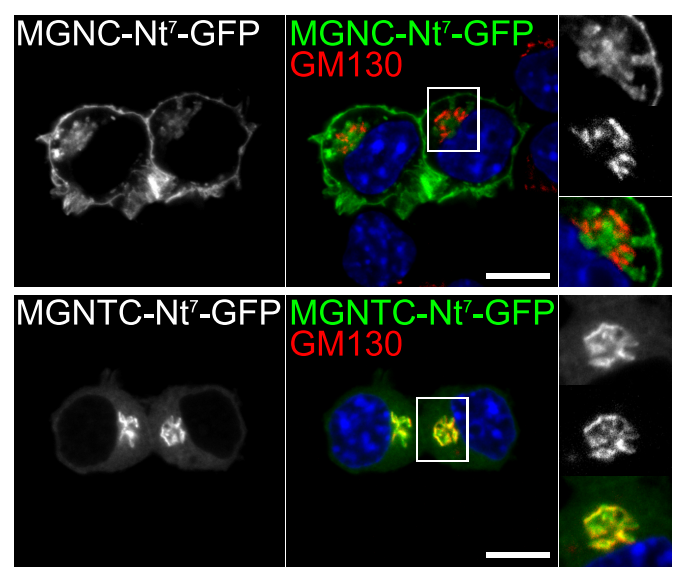

e

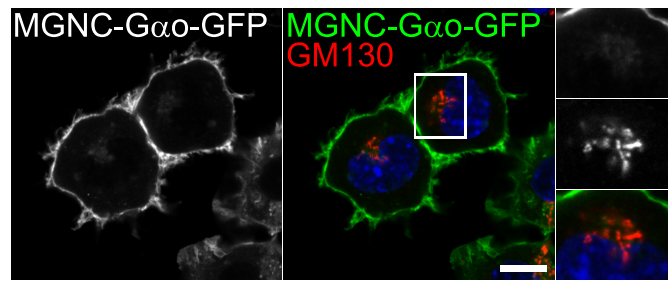

h

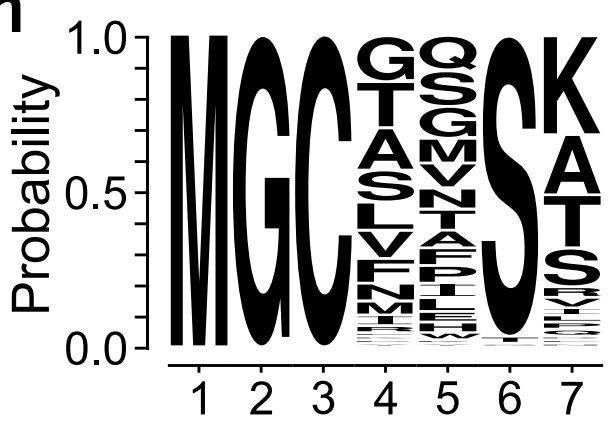

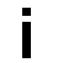
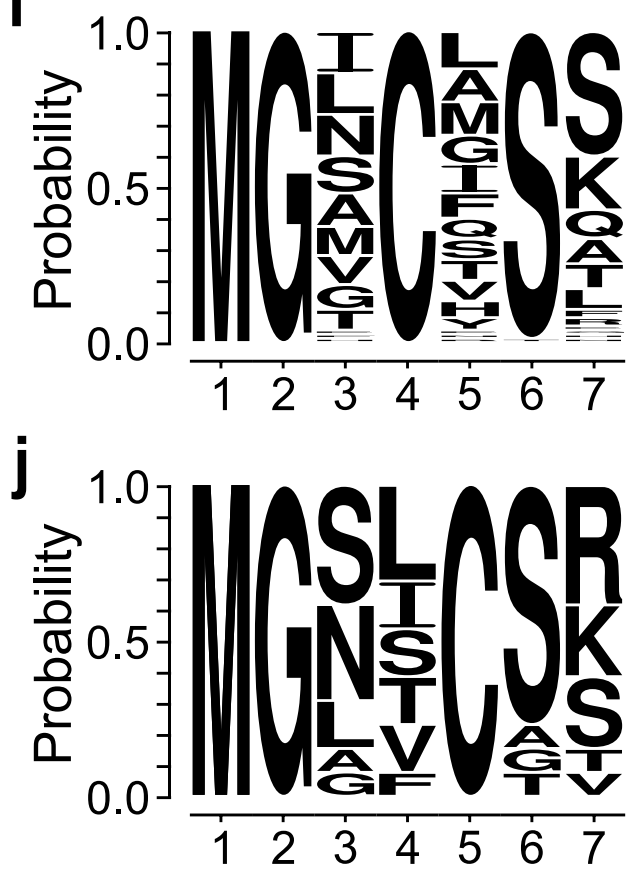

b

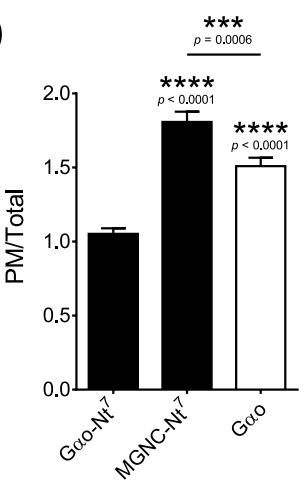

f

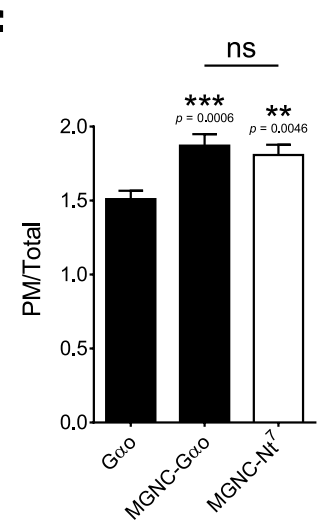

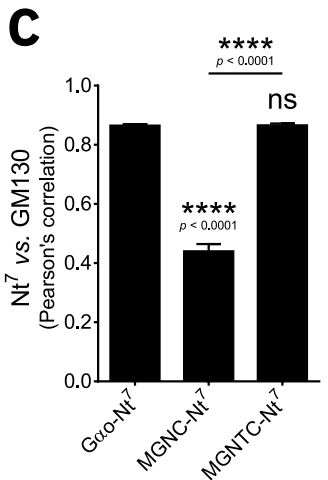

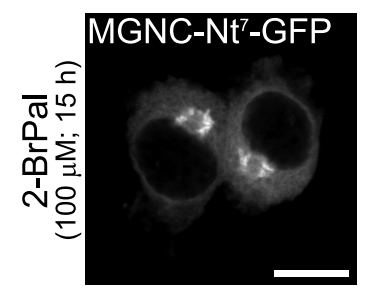

k

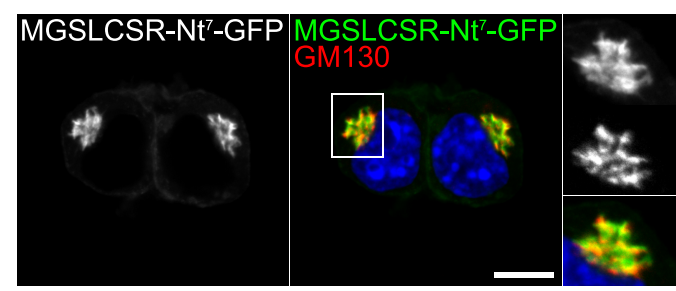

m

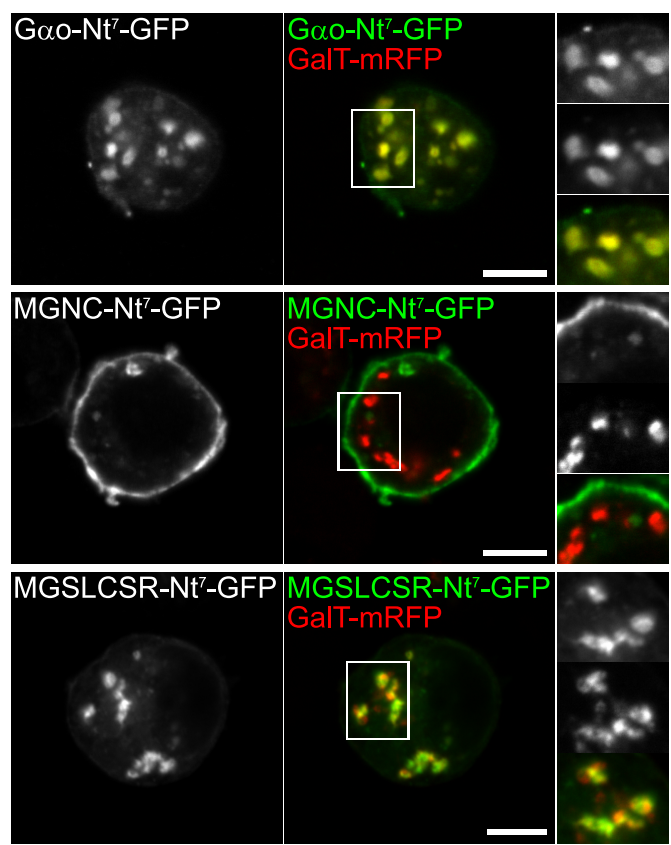

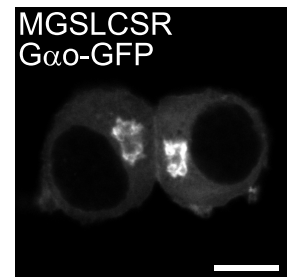

Figure 2_Solis et al. 
bioRxiv preprint doi: https://doi.org/10.1101/2020.08.25.266692; this version posted August 26, 2020. The copyright holder for this preprint

(which was not certified by peer review) is the author/funder, who has granted bioRxiv a license to display the preprint in perpetuity. It is made available under aCC-BY-NC-ND 4.0 International license.
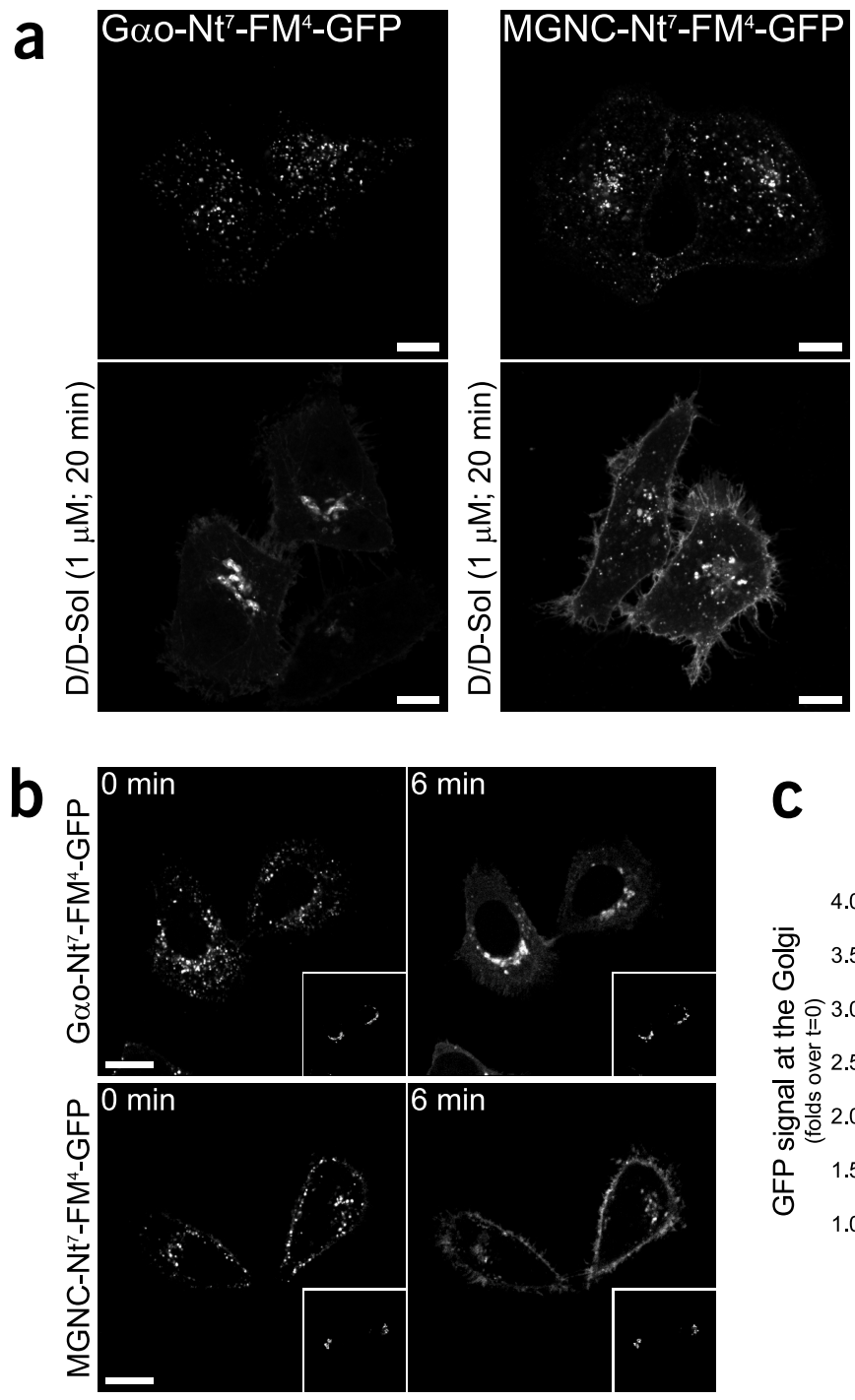

C
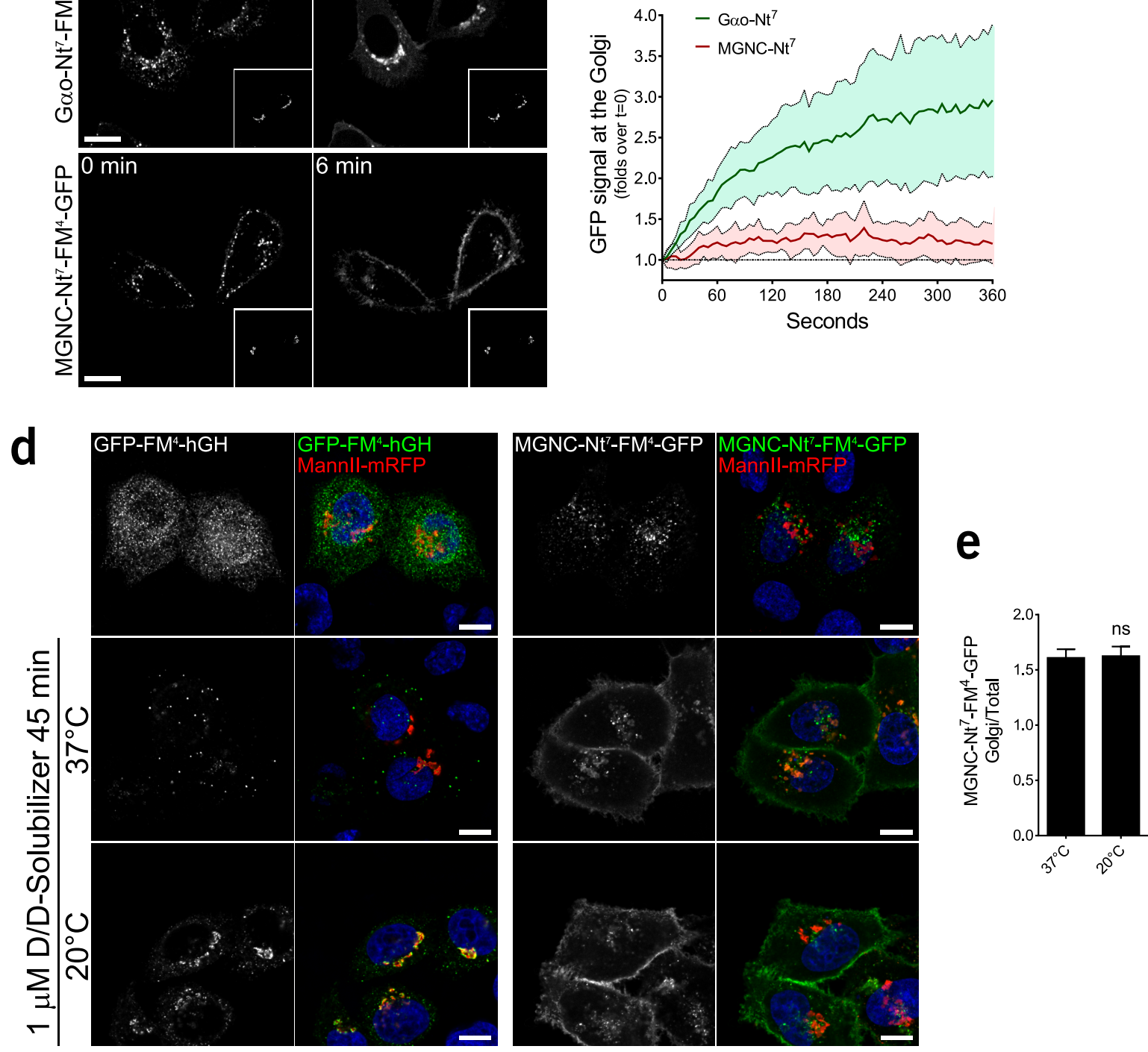

Figure 3_Solis et al. 
bioRxiv preprint doi: https://doi.org/10.1101/2020.08.25.266692; this version posted August 26, 2020. The copyright holder for this preprint (which was not certified by peer review) is the author/funder, who has granted bioRxiv a license to display the preprint in perpetuity. It is made available under aCC-BY-NC-ND 4.0 International license.

a

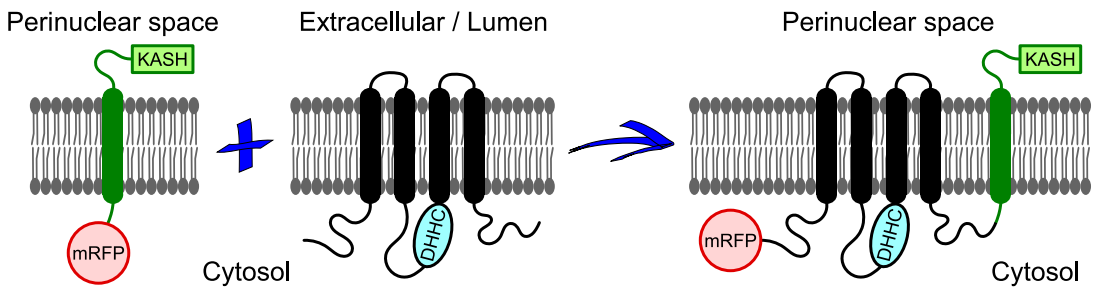

b
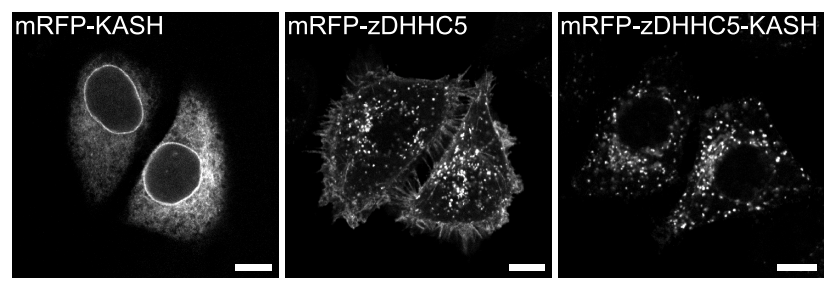

C
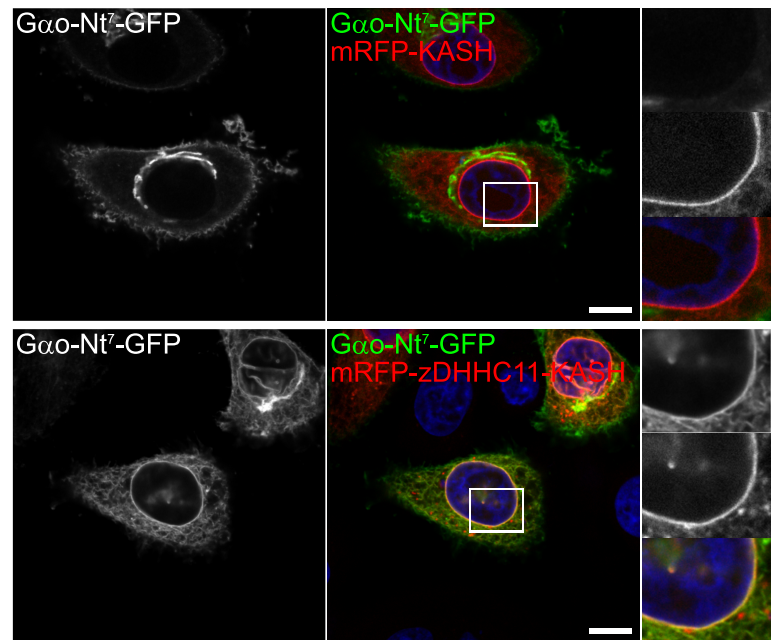

d

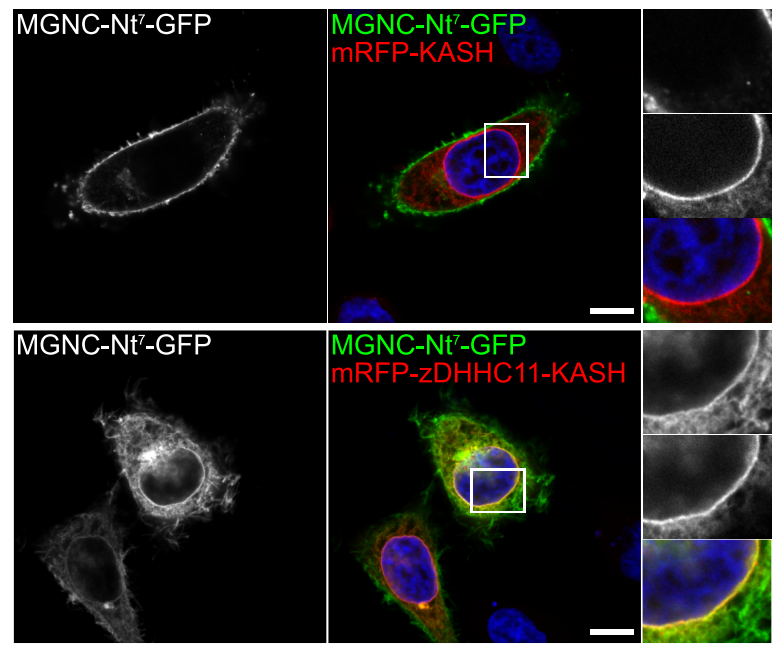

e

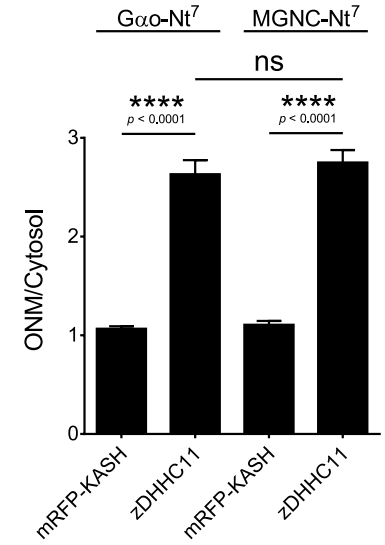

h

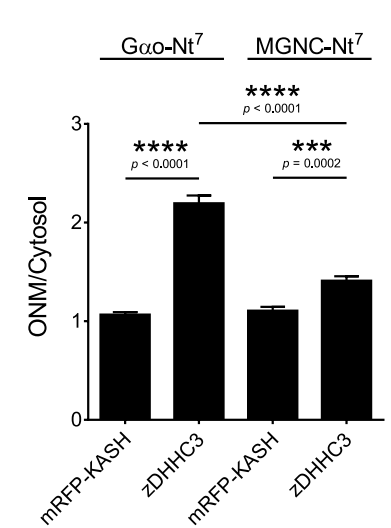

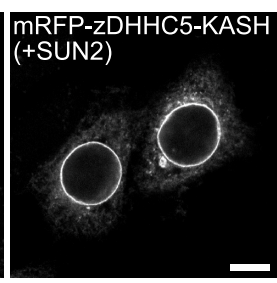

f
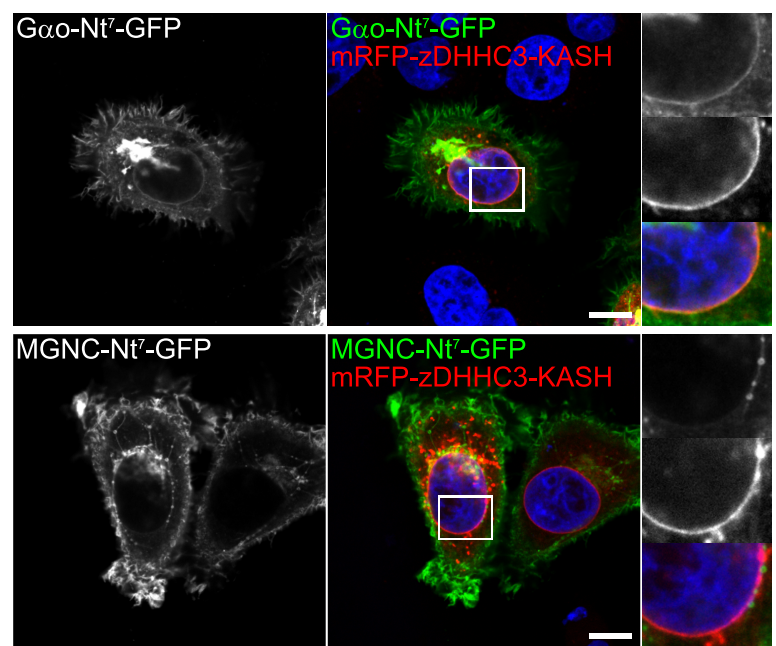

g
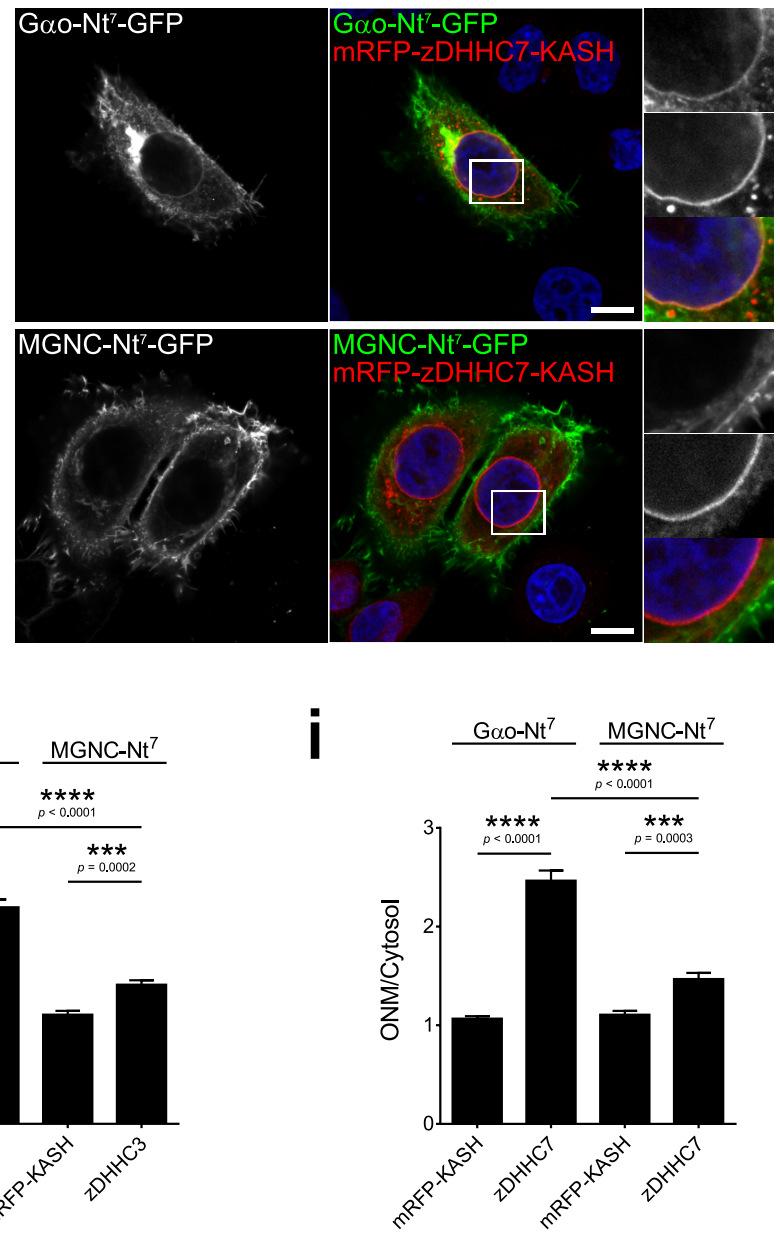

Figure 4_Solis et al. 
bioRxiv preprint doi: https://doi.org/10.1101/2020.08.25.266692; this version posted August 26, 2020. The copyright holder for this preprint (which was not certified by peer review) is the author/funder, who has granted bioRxiv a license to display the preprint in perpetuity. It is made available under aCC-BY-NC-ND 4.0 International license.
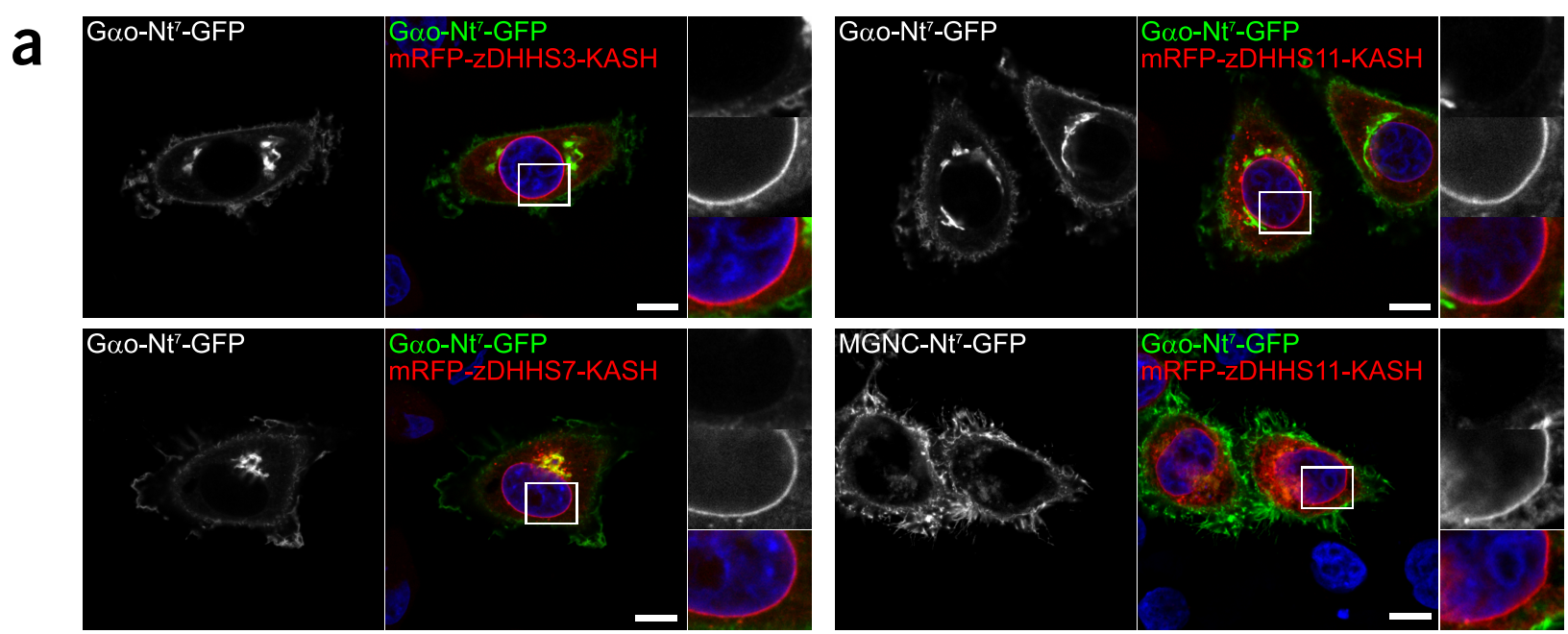

b

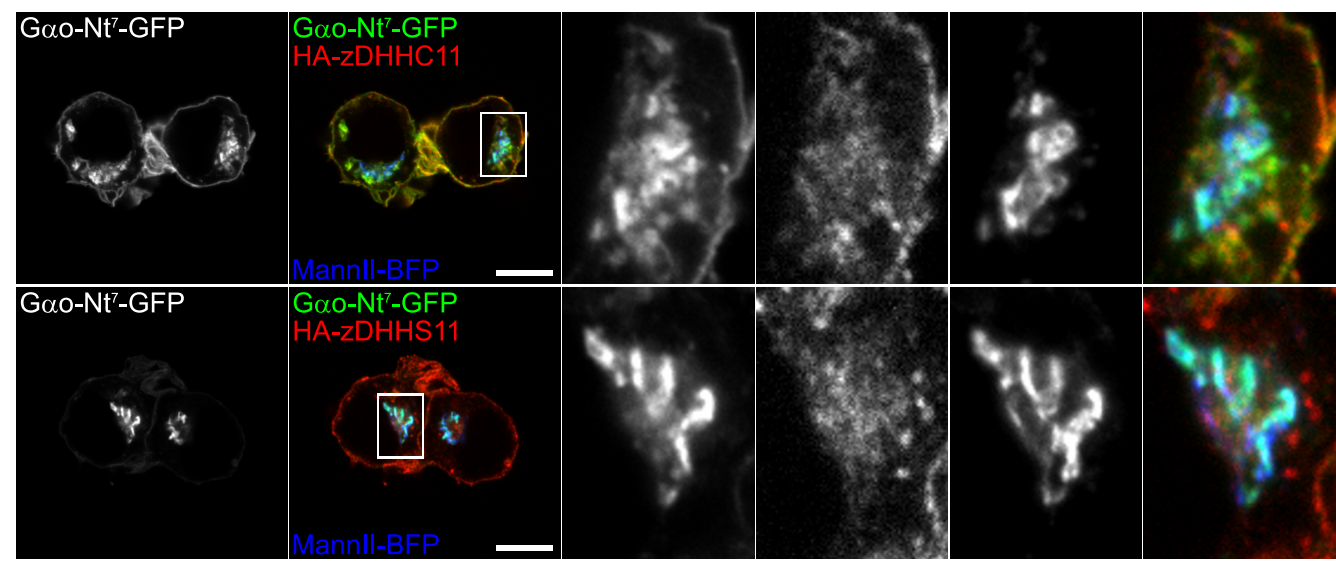

C

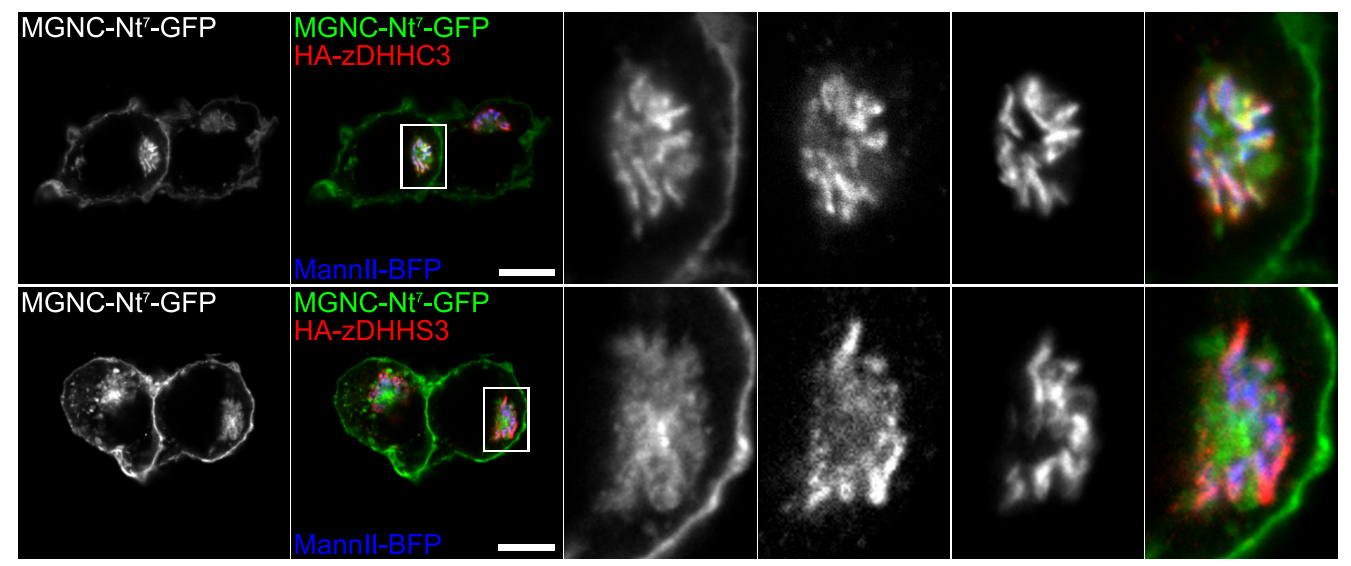

d

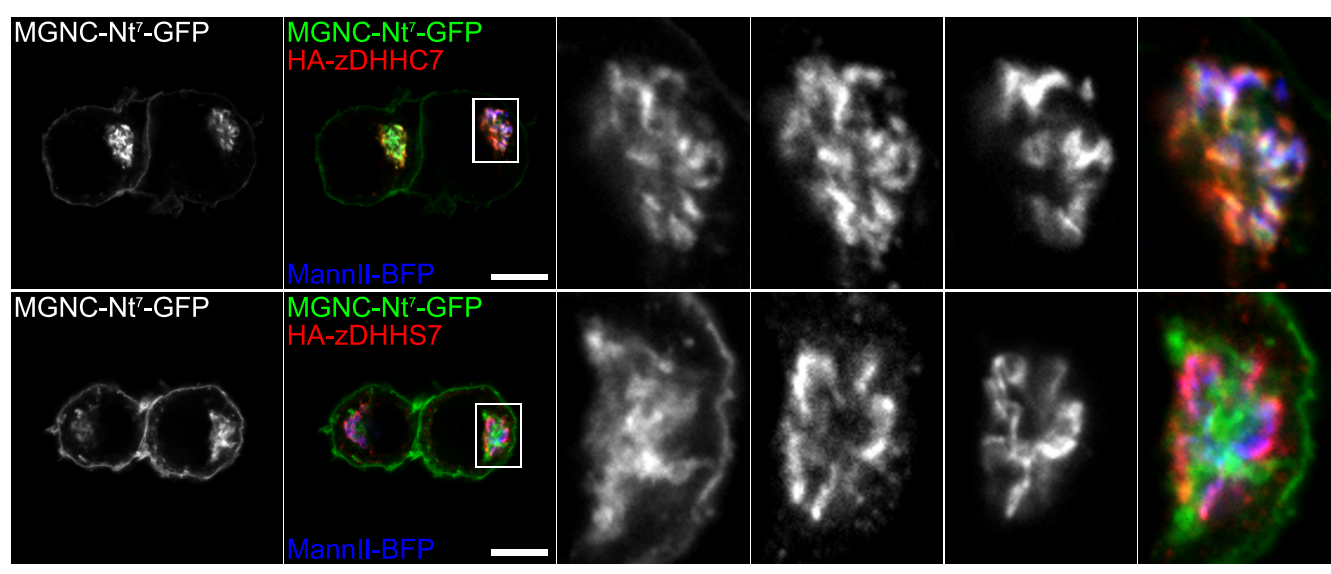

e

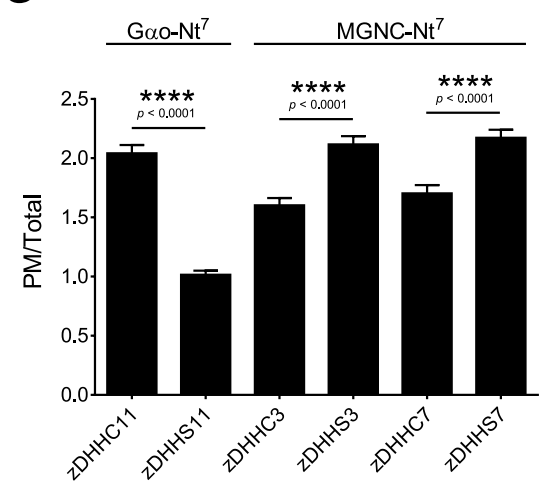

$\mathbf{f}$

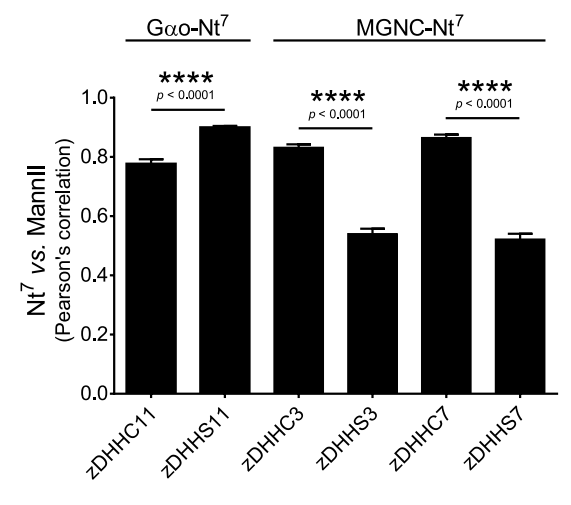


bioRxiv preprint doi: https://doi.org/10.1101/2020.08.25.266692; this version posted August 26, 2020. The copyright holder for this preprint (which was not certified by peer review) is the author/funder, who has granted bioRxiv a license to display the preprint in perpetuity. It is made available under aCC-BY-NC-ND 4.0 International license.

\section{a}
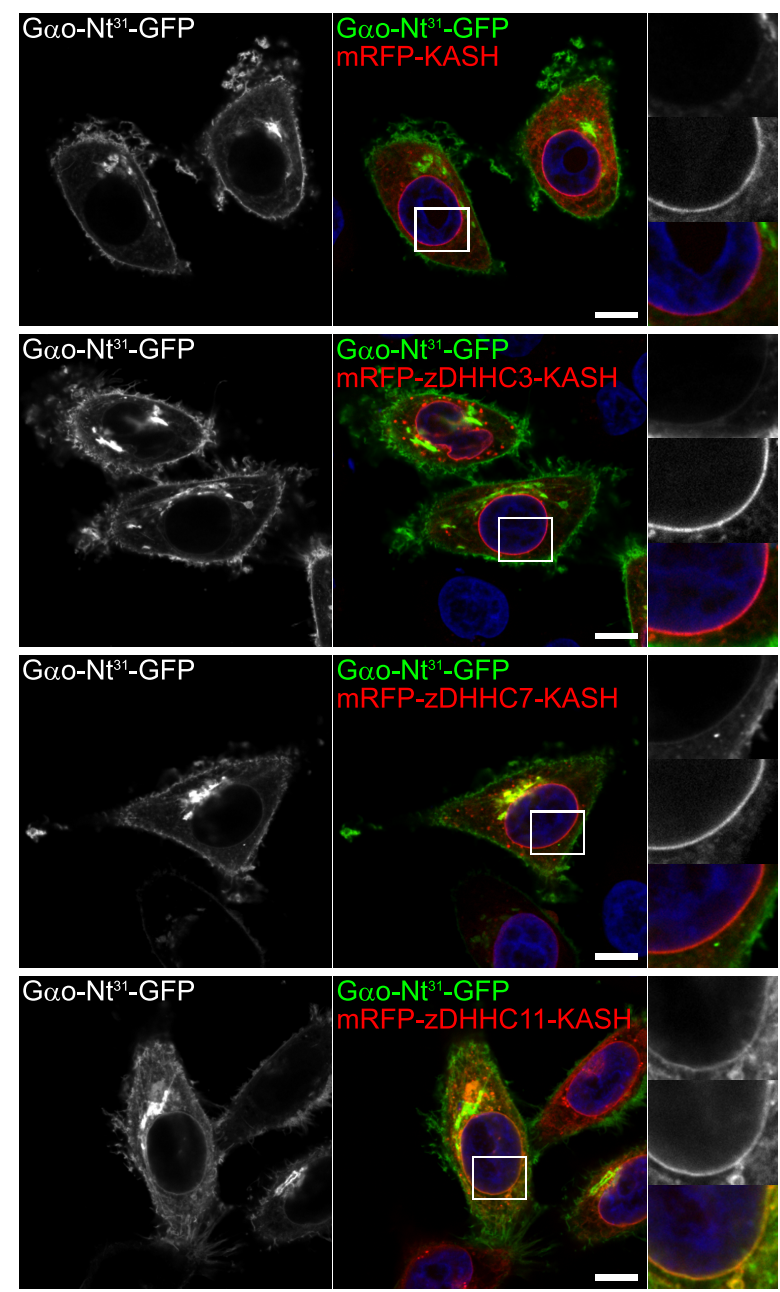

b

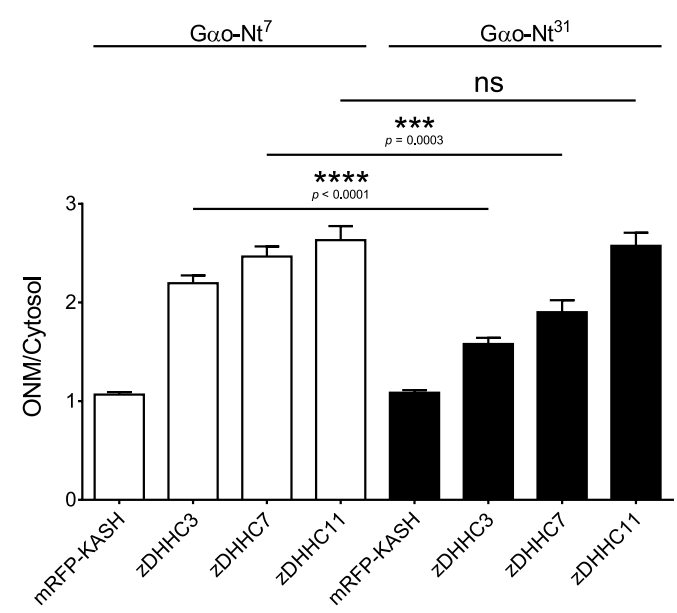

\section{C}
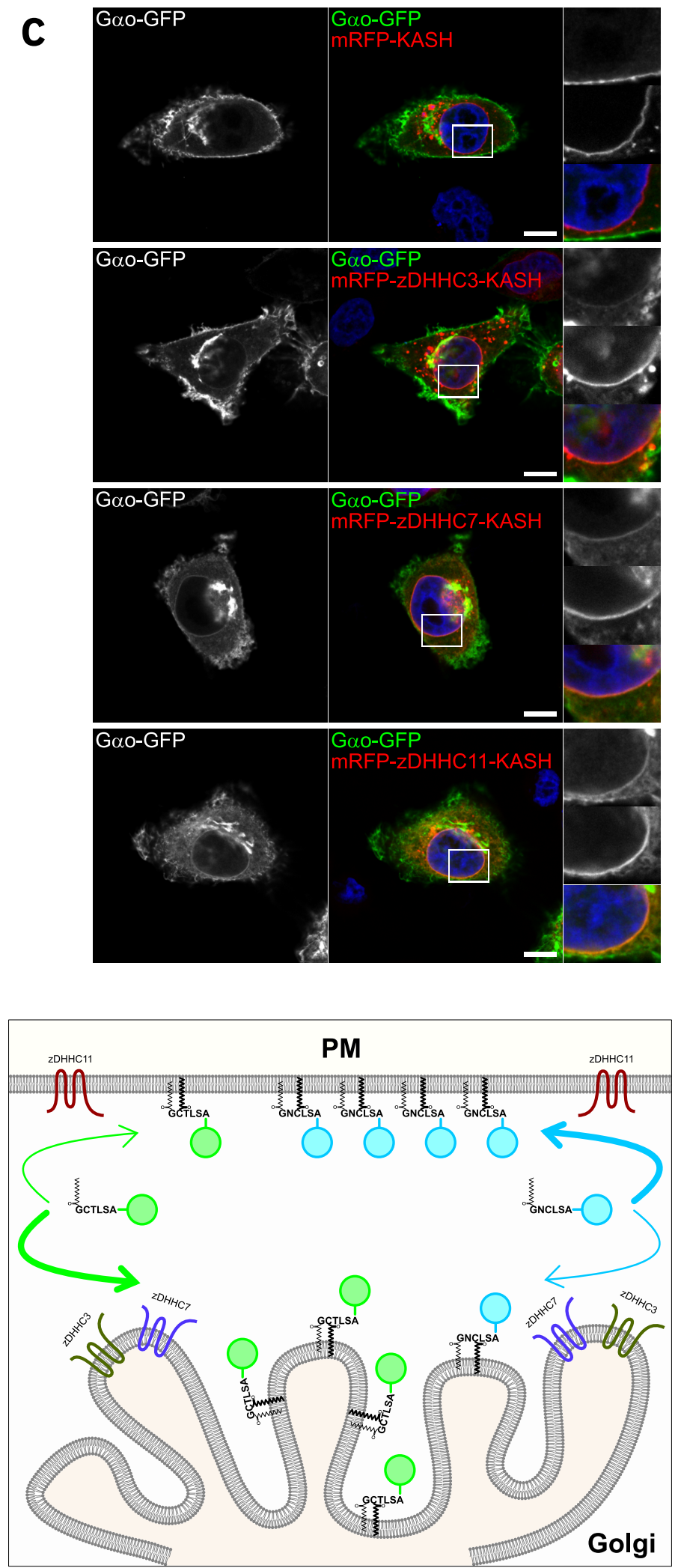